\title{
Efficient frontiers in a frontier state: Viability of mobile dentistry services in rural areas
}

\section{Authors: Andreas H. Thorsen and Ronald G. McGarvey}

NOTICE: this is the author's version of a work that was accepted for publication in European Journal of Operational Research. Changes resulting from the publishing process, such as peer review, editing, corrections, structural formatting, and other quality control mechanisms may not be reflected in this document. Changes may have been made to this work since it was submitted for publication. A definitive version was subsequently published in European Journal of Operational Research, v. 268, no. 3, August 2018.

DOI\# 10.1016/i.ejor.2017.07.062

Thorsen, Andreas H., and Ronald G. McGarvey. "Efficient frontiers in a frontier state: Viability of mobile dentistry services in rural areas." European Journal of Operational Research 268, no. 3 (August 2017): 1062-1076. DOI: 10.1016/j.ejor.2017.07.062.

Made available through Montana State University's $\underline{\text { ScholarWorks }}$ scholarworks. montana.edu 
Efficient Frontiers in a Frontier State: Viability of Mobile Dentistry Services in Rural Areas

\author{
Andreas Thorsen ${ }^{\mathrm{a} *} \quad$ Ronald G. McGarvey ${ }^{\mathrm{b}}$

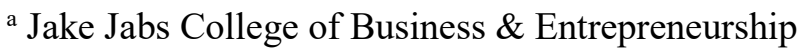 \\ Montana State University \\ 350 Jabs Hall \\ P.O. Box 173040 \\ Bozeman, Montana 59717-3040, USA. \\ * Corresponding author: andreas.thorsen@montana.edu \\ ${ }^{\mathrm{b}}$ Department of Industrial and Manufacturing Systems Engineering, and Truman School of Public \\ Affairs \\ E3437 Thomas and Nell Lafferre Hall \\ University of Missouri \\ Columbia, Missouri 65221, USA. \\ mcgarveyr@missouri.edu
}




\begin{abstract}
This study investigates the implications of adding mobile dentistry services to a community health center $(\mathrm{CHC})$ in a rural area. $\mathrm{CHCs}$ are not-for-profit healthcare organizations which provide comprehensive primary care services to patients in the US, primarily for under-served and uninsured populations. We estimate the demand for the service in a five-county region in southwestern Montana, USA and work with stakeholders to determine a set of potential service locations. A mixed-integer optimization model is formulated to determine the frequency of stops in each location over a finite (six month) planning horizon with the goal of improving accessibility and availability of dental services while maintaining financial sustainability of the $\mathrm{CHC}$. The financial considerations and social impact of offering a mobile dentistry service in southwestern Montana are assessed. Computational results based on a case study demonstrate the challenges facing mobile dentistry operations to increase access to under-served populations in a financially viable manner. Hybrid solutions, in which care is offered at a mix of fixed locations and mobile locations, appear to best balance the objectives of financial sustainability and expanded access to care.
\end{abstract}

\title{
Keywords
}

OR in health services, Community operational research, Network design, Location selection, Mobile dentistry, Community-based operations research

\section{Introduction}

Dental health is essential for overall general health and well-being (Davis et al., 2010). The Surgeon General's 2000 report notes that oral infections are associated with increased risk of diabetes, heart disease, and stroke (US Department of Health and Human Services, 2000). In addition to the connection between oral health and systemic health, it has been established that oral disorders have an adverse effect on quality of life and economic productivity (Hollister and Weintraub, 1993).

Access to dental care is a major issue for those in rural areas in the US, where the closest dental office may be far from a person's home (US Department of Health and Human Services, 2004). This dental access problem is magnified for people who do not have dependable travel options (e.g., in areas without public transportation people may need to borrow a car or obtain a ride from a family or friend). There are also financial barriers to dental care access, since many people without dental insurance cannot afford to pay for care out-of-pocket. Medicaid is a public insurance program for low-income people in the US that includes coverage for dental services in some states (Medicaid.gov, n.d.). However, Medicaid-enrolled patients may not be able to easily find a dental provider willing to accept their public insurance. A major access point for such patients is at community health centers (CHCs), which are not-for-profit healthcare organizations providing comprehensive primary care services to 
people including those who are medically under-served and uninsured. However, especially in rural areas, there can still be a large distance from a person's home to the nearest CHC.

Mobile dentistry has emerged as an alternative service delivery method to counteract these access problems. Mobile dentistry involves dentists, dental hygienists, and dental assistants traveling in vans or trailers with onboard dental equipment to serve patients at locations near the patients' homes. A challenge for organizations considering mobile dentistry as a viable business model is the balancing act of extending services to as many people as possible in a financially sustainable way. Scholarly discourse has not adequately addressed this problem.

In this paper, we develop a data-driven, optimization-based approach to explore the potential for a rural $\mathrm{CHC}$ to add mobile dentistry services, and we apply the model to a five-county region in southwestern Montana. We consider the network design of the mobile dentistry operation, determining the location of mobile dentistry stops, the frequency of visits, and the composition of the dental care team over a six-month planning horizon. We model the mobile dentistry network design problem (MDNDP) as a mixed-integer program, with the goal of improving dental care accessibility and availability for under-served populations while considering the financial sustainability of the service.

This paper makes several contributions to research on mobile dentistry. First, we use a communitybased operations research (CBOR) approach to identify the problem and formulate the MDNDP as a mixed-integer program to improve accessibility and availability of dental services while maintaining financial sustainability. Next, we use a data-driven, optimization-based methodology to answer questions regarding how a local CHC could improve their rural dental mission in an economically sustainable fashion. Elsewhere, this research could be generalized and leveraged by healthcare organizations in rural regions to understand the tradeoffs regarding the operation of mobile healthcare facilities providing other types of service (e.g., optometry).

The remainder of this paper is structured as follows. The next section presents a literature review related to rural dental service access, mobile dentistry, and mobile facility routing more generally. Section 3 illustrates the research process used to identify and formulate the model, and presents the model. Section 4 discusses the application of our model to a case study in southwestern Montana and presents the results, followed by a discussion in Section 5. Section 6 presents concluding remarks.

\section{Problem description}

\subsection{Dental needs in rural areas}

Skillman et al. (2010) describe issues facing rural populations which include lower utilization of dental care services, and higher rates of cavities and permanent tooth loss compared to urban populations. The authors suggest that reasons for these differences include the lower rate of dental insurance coverage, less access to dental services, and higher poverty among those living in rural areas. The US Department of Health and Human Services (2004) identifies several factors contributing to 
access problems for dental services in rural areas, including: lack of fluorinated water supplies, geographic isolation, inadequate transportation, higher rates of poverty, larger percentage of elderly people, low rates of dental insurance, acute provider shortages, and difficulty finding providers willing to treat Medicaid-enrolled patients. Kimminau and Wellever (2011) introduce the concept of "Dental Service Area Deserts" to describe rural areas that don't have coverage from a primary care dental office.

These problems in rural areas are intensified for the elderly, as older adults in rural areas have less favorable oral health and are less likely to be insured than their urban counterparts (Vargas, Yellowitz, and Hayes, 2003). Traveling for services may be especially difficult for some populations, including the elderly. Access to dental care is a prevailing problem for 1.75 million people in long-term care facilities in the US (Guay, 2005).

Mobile dentistry has emerged as an approach to address these access problems. For example, Apple Tree Dental ${ }^{1}$ is a non-profit organization in Minnesota that operates a mobile dentistry service for group homes, schools and other locations. Apple Tree Dental's business model involves bringing portable equipment to locations, such as childcare centers, and temporarily transforming them into dental offices.

Another approach is for dentists to travel in vans or trailers with onboard dental equipment to serve patients. An example of this type of operation is the Ronald McDonald Care Mobile (RMCM) that operates out of Billings, Montana and receives financial support from St. Vincent's Healthcare Foundation (Ronald McDonald Care Mobile, 2016). RMCM operates a 40-foot camper plus truck cab with two chairs, its dental operations are staffed by a dentist and a dental hygienist. It currently serves 43 schools for dental screenings, and visits approximately 25 towns for pediatric clinical work. According to their dental hygienist, RMCM visits one location each day they operate, operating up to two days per week with a maximum trip distance of 120 miles (M. Utley, personal communication, May 24, 2016). While it brings tremendous increase in access for pediatric patients throughout Montana, RMCM would not be financially sustainable without charitable donations and grant funding.

Another example of mobile dentistry in Montana is the operation at Granite County Medical Center (GCMC) (Briggeman, 2013). GCMC was able to purchase a mobile dental van due to winning a grant from the Health Services and Resources Administration in 2010. However, the mobile operation was not sustainable and was discontinued after one year. Despite many examples available of mobile dentistry being supported by grants, it is an open question of under what conditions mobile dental operations for under-served populations in rural areas can be financially sustainable on their own.

\subsection{Academic literature on mobile facility location}

There is an extensive stream of research literature on mobile facility location. Hodgson, Laporte, and Semet (1998) propose a covering tour model to develop routes for a mobile health care facility in Ghana, Africa. Lei, Lin, and Miao (2014) examine a mobile facility routing and scheduling problem

\footnotetext{
${ }^{1}$ Apple Tree Dental mobile dentistry website: http://www.appletreedental.org/services/mobile-dental-care/
} 
under stochastic demand and formulate the problem as a two-stage stochastic programming model. The authors mention applications including trucks equipped with cellular base stations to serve areas where cell networks are disrupted, and fast food service from vans. Lei, Lin, and Miao (2016) develop a twostage robust optimization approach for mobile facility fleet sizing and routing under demand uncertainty using a network flow formulation. The models from the previous three papers mentioned do not include setup times, which is a significant operational constraint for the mobile dentistry problem discussed in this paper. In a related paper that does include setup times, Doerner, Focke, and Gutjahr (2007) develop tours for mobile healthcare facilities in a developing country by modeling the problem as a variant of a vehicle routing problem. However, the mobile dentistry problem studied in this paper involves longer setup times than the ten minute setup assumed by Doerner et al. (2007). Other mobile dentistry operations in Montana cite setup times of around 30-45 minutes, with similar breakdown times (M. Utley, personal communication, May 24, 2016). Therefore, for the application considered in this paper, it is assumed to be only practical to visit one location on a given day, leaving from and returning to the home location each day, which eliminates the need for inclusion of routing constraints. Other distinctions of the current paper that have not been addressed in the literature are the consideration of financial sustainability and equity of service across locations for mobile dentistry.

\subsection{Approaching the problem using a CBOR approach}

This research problem falls squarely within the realm of CBOR as it highlights "the needs and concerns of disadvantaged human stakeholders" (Johnson and Smilowitz, 2012). Johnson and Smilowitz (2012) remark that the defining characteristics to distinguish CBOR from other areas of public-sector OR are the focus on human stakeholders, disadvantaged populations, and communitylevel characteristics such as socio-economic status. They also mention that the defining characteristic of CBOR problems is the involvement of multiple stakeholders and multiple decision makers. In our problem, these groups include the community partner considering offering a mobile dentistry service, individuals in rural communities who may benefit from the mobile dentistry service and, indirectly, dental service providers who may be impacted by a mobile operation in proximity to their service area, and the donors (as CHCs obtain a large portion of their revenue from government grants and private donations). CBOR, which originated in the US and was first termed by Johnson and Smilowitz (2007), is related to community operational research (COR) which originated in the UK over thirty years ago. Indeed, both CBOR and COR are usually concerned with the needs of "disadvantaged, underrepresented, and underserved populations" (Johnson, Midgley, and Chichirau, 2017). While both CBOR and COR emphasize meaningful stakeholder/community engagement by definition, the main difference is that COR involves "an awareness that engagement drives the choice of problem-solving approach and methods" (Midgley, Johnson, and Chichirau, 2017). Further, direct engagement with local communities is viewed as a necessary condition for COR, whereas stakeholder engagement is the requirement for $\mathrm{CBOR}$. 
Several papers in the CBOR literature are related to ours. For example, Hindle and Vigden (2017) adopt an action research framework to help a UK charity that runs a foodbank network develop their analytics strategy. They used a combination of geo-spatial data (specifically, open-source Census data on poverty and the charity's own data) to develop predictive demand models. A seminal paper in the CBOR literature, Johnson (2006), uses a framework of value-focused thinking and optimization to select sites for community correction centers. Sefair, Molano, Medaglia, and Sarmiento (2012) develop a multiobjective optimization model for locating new urban parks using geographic information system (GIS) data. The research process undertaken for this study is related to the last two papers, involving the collection of hard and soft data from several stakeholder groups (e.g., practitioners and governmental) as well as analysis of GIS data, and finally formulation of an optimization model to give decision makers deeper insight into the impact of various operational policy decisions. The research process is described in more detail in the next section.

\section{Research process}

The study began when a CHC operating in southwestern Montana, Community Health Partners (CHP) asked the lead author to examine the need for and the financial viability of a mobile dentistry service in their service region. CHP is highly regarded in southwestern Montana and seeks to increase access to healthcare for the improvement of the community. The organization operates several medical and dental clinics, and provides educational support services. CHP serves primarily uninsured and under-insured patients, offering sliding scale fees based on income, and they seek grants to supplement public insurance program reimbursements.

The nature of the engagement with our main community partner, CHP, was related to problem identification, problem formulation, and solution. Through many early discussions, the nature of the problem was unearthed (i.e., uninsured and under-insured adults who are geographically isolated and therefore do not receive dental care) and the financial viability of mobile dentistry for improving the status quo was unknown. Then, CHP was involved with problem formulation in order to ensure that our model and its assumptions reflect reality as close as possible. Besides sharing extensive data used to parameterize the model, CHP helped with other aspects of problem formulation including: determining the composition of the mobile dentistry team; determining the service region; choosing the set of towns visited.

We collected and analyzed data from several different sources. Quantitative data was collected from sources such as the US Census, and GIS databases. Other stakeholders besides CHP responsible for public health in the region were engaged in order to help identify the problem and formulate the model. Since CHP does not currently operate a mobile clinic, we engaged several mobile clinics who currently operate or previously operated in Montana to ensure the modeling assumptions were reasonable. Data were collected by engaging several other stakeholder groups including: professional organizations in 
Montana, including the Montana Dental Association; governmental entities such as Montana Office of Rural Health and Area Health Education Center (ORH-AHEC), and Montana Department of Public Health and Human Services (DPHHS). Philosophically, in alignment with CBOR principles, this research was conducted under the assumption that interacting with organizations who are trying to solve the problem of providing rural dental services is necessary to successfully formulate models that are applicable to the locality so that the recommendations presented to the decision makers are specific, valid, useful, and capture the differing perspectives and objectives of the many interested parties.

The following were the key steps of our research process:

(a) Data analysis

(b) Discussion with stakeholder groups

(c) Model development

(d) Solution and sensitivity analysis

(e) Policy recommendations and conclusions

Several iterations of steps (a), (b), and (c) were conducted as various stakeholder groups were engaged in the process. First, leaders of CHP identified the five-county region consisting of Gallatin, Park, Meagher, Sweet Grass, and Broadwater counties in Montana as their intended service region. The counties are part of a unique setting in the state of Montana, as the state has the second-lowest population density of any state in the US of 6.8 people per square mile (US Census Bureau, n.d.). Montana and four other states in the US are federally designated as "Frontier States" since 50\% of their counties have an average population density of under six people per square mile (Rural Health Information Hub, n.d.). Further, four of the five counties considered in our study have even lower population densities than the state average. The population and size of the five-county region we consider in this paper is shown in Table 1.

Table 1.

Descriptive population and size information about the five-county region in our study, according to the 2010 US Census

\begin{tabular}{lccc}
\hline County & Population & $\begin{array}{c}\text { Land area } \\
\text { (sq. mi.) }\end{array}$ & $\begin{array}{c}\text { Population density } \\
\text { (average per square mile } \\
\text { of land) }\end{array}$ \\
\hline Gallatin & 89,513 & 2,602 & 34.4 \\
Park & 15,636 & 2,803 & 5.6 \\
Broadwater & 5,612 & 1,192 & 4.7 \\
Sweet Grass & 3,651 & 1,855 & 2 \\
Meagher & 1,891 & 2,391 & 0.8 \\
\hline
\end{tabular}

We define the under-served population as economically disadvantaged adults who either have no insurance or are enrolled in Medicaid, who also lack access to existing services due to the large distance 
to the nearest clinic. Children are not included in our analysis, since there is not an unmet need in the region according to several stakeholders. This is because it is common for Medicaid providers to accept only children with Medicaid insurance as patients, while not being willing to serve adults due to the lower reimbursement rates. In Montana, dental services covered for Medicaid-enrolled adults includes preventative services in addition to an annual cap of $\$ 1,225$ for treatment services (Montana Department of Public Health and Human Services, n.d.).

\subsection{Estimating the under-served population's demand for mobile dentistry services}

In order to obtain the geographic distribution of the under-served population of interest, we analyzed US Census data at the most disaggregated level available using the GIS software ArcMap 10.3.1. This allowed us to identify regions with high populations of economically disadvantaged adults who either had no insurance or were enrolled in Medicaid.

Data come from the US Census American Community Survey (ACS) 2014 5-year estimate. We examined data for Census Block Group level counts of people with different types of health insurance coverage for several age ranges. In the current study we leverage these data to create a measure of the number of under-served individuals in a given Census Block using three different insurance types: uninsured (all adults), patients who only have Medicare (ages 65+), and Medicaid patients (all adults). This measure captures our aggregate demand set in this region.

To measure the number of uninsured adults in each Block Group, first we sum the counts 'No Health Insurance Coverage' for age groups '18-34 Years', '35-64 Years', and '65 Years and Over' (n=13,942). We use data on lack of health insurance coverage as a proxy for lack of dental insurance. Unfortunately, to the best of our knowledge, there are no datasets for dental insurance coverage of small geographic areas. Nevertheless, we claim that our data represent a conservative approximation of the number of people without dental insurance. In support of this claim, in 2012 almost half of Americans were without dental insurance compared to $15 \%$ without medical insurance (Alliance for Health Reform, 2012).

Another public insurance program we consider is Medicare. Medicare, a program for people who are 65 or older, does not cover dental care in general. Therefore, we also include the counts of 'With Medicare Coverage Only' for age group '65 Years and Over' and 'With Direct-Purchase and Medicare Coverage' for age group '65 Years and Over', to capture elderly adults who do not have dental insurance. Kiyak and Reichmuth (2005) remark that estimates of rates of dental insurance for older people in the US range from 14.5 to 28.4 percent. Based on this, in order to approximate the number of people without dental insurance we reduce the counts of 'With Medicare Coverage Only' and 'With Direct-Purchase and Medicare Coverage' by $30 \%$ (from $n=7,752$ to $n=5,466$ ), in order to exclude those estimated $30 \%$ of individuals who may have purchased dental insurance directly. Taking these two measures together, the total number of uninsured adults in the five-county region is estimated to be 19,918 . 
To measure the number of Medicaid-enrolled adults in each Block Group, first we sum the counts 'With Medicaid/Means-Tested Public Coverage Only' for age groups '18-34 Years' and '35-64 Years' and the count of 'With Medicare and Medicaid/Means-Tested Public Coverage' for age group '65 Years and Over' $(\mathrm{n}=2,471)$.

Next, the granularity level of detail of our data is considered. Data were collected at the secondsmallest unit of analysis for US Census data, the Block Group level, which includes 600 to 3000 people per geographic unit. Block Groups can be quite geographically large for rural areas. For our five-county region, the number of Block Groups per county are as follows: Gallatin (60), Park (17), Sweet Grass (5), Broadwater (4), and Meagher (2). This level of aggregation is not sufficiently detailed for our analysis. The smallest unit of analysis in the US Census is called the Census Block (there are 2,048 Blocks in this five-county region). In order to estimate the number of uninsured adults and Medicaidenrolled adults at a finer level of geographic detail, we assume that Blocks are homogenous within any Block Group for our data. Therefore, the distribution of demand within each Block is assumed to be the same as the distribution of demand of its Block Group. Using information on the total population of each Block Group from the 2010 US Census we distribute Block Group level data to each Block according to the proportion of the Block Group population living in the Block. Figure 1 illustrates our estimated distribution of total uninsured adults and Medicaid enrollees over the five-county region in a heat map.

\subsection{Estimating the existing supply of dental services for under-served populations in the region}

It is important to determine the existing dental services available for the under-served adult population in the region to ensure that demand is not over-estimated. To do so, we obtained a list from the DPHHS website of 41 private practice dentists who are enrolled as Medicaid providers in the fivecounty region (Montana Department of Public Health and Human Services, 2016). We assume that dentists enrolled as Medicaid providers and federally-funded CHCs are the only access points for Medicaid-enrolled adults, the latter of which has only two locations in the region, with patient demand far exceeding staffing levels. Thirty-five of the 41 Medicaid providers operate in Gallatin County. Of the six remaining, five operate in Park County, and one in Broadwater County. There are no Medicaid providers in Sweet Grass County or Meagher County. Also, all five Medicaid providers in Park County operate in the same location (Livingston), suggesting the possibility of access problems for people living in poverty in Park County who live far away from this location.

In order to have a more accurate estimate of the supply of services for the under-served adult population, we conducted a content analysis. Specifically, we visited the websites for each of these 41 dentists, and we removed the dentist from our sample if there is indication that they do not serve adult Medicaid enrollees. Reasons for removing dentists from the sample were as follows: One dentist moved their practice to Idaho, an hour from the Montana border; two dentists were clearly pediatric dentists, and did not serve adults; two dentists' practices were permanently closed; one dentist had a policy of 
accepting several private insurance policies in addition to stating, "We also accept Medicaid for children." We assumed this dentist did not serve adult Medicaid patients, so the dentist was removed from the sample. Twelve dentists did not have websites, and therefore were left in the sample. In our final sample we have 30 dentists in Gallatin County who potentially serve Medicaid patients, five dentists in Park County, and no dentists in the remaining three counties in our study.

Furthermore, we learned, through discussions with the DPHHS Oral Health Program Coordinator, that many private practice dentists who are enrolled as Medicaid providers (i.e., the 35 dentists remaining in the sample) may actually treat very few Medicaid patients, or may only treat Medicaidenrolled children, and that there is an indication that a large gap exists between the estimated number of adult Medicaid-enrollees in the five-county region and the actual amount of services rendered ( $\mathrm{T}$. Hollingsworth, personal communication, May 2, 2016). This supports our assertion that dental service access is a problem in the region.

\subsection{Identifying CHP's list of mobile dentistry stops}

The set of potential stops considered were all cities, towns, and census-designated places (CDPs) that were identified in the 2010 Census in the region. These are the areas with settled population concentrations, including incorporated places that have a legal description, and unincorporated places recognized by the US Census as places identified by name with a settled population but no legal status or elected officials (US Census Bureau, n.d.). In the five-county region, 43 such places were identified, with populations ranging from 27 people (in Toston) to 37,280 (in Bozeman). The complete list of places is presented in Table A.1 in Appendix A.

In order to obtain information about the geographic distribution of the under-served population of interest, we analyzed US Census data at the most disaggregated level available (Census Blocks) using the GIS software ArcMap 10.3.1. This allowed us to identify regions with high populations of economically disadvantaged adults with either no insurance or enrolled in Medicaid. We created maps to visualize these data.

We also analyzed the poverty in the region, using Census data from the 2014 ACS on the counts of households in each Block Group who earn less than $\$ 20,000$ of household income in the past year $(n=8,067)$. Then, using data on the total population from the 2010 US Census, we distributed Block Group level data to each Block according to the proportion of the Block Group population living in the Block. The cut-off of $\$ 20,000$ in past-year household income was chosen as a conservative estimate of poverty status, as we did not have data on the number of people in each household. Based on the 2016 Federal Poverty Limit guidelines (HealthCare.gov, 2016), this corresponds to under 200\% of FPL for a single person, under $133 \%$ of FPL for a family of two, and under $100 \%$ for a family of three or more. Figure 1 illustrates the distribution of households earning less than $\$ 20,000$ in the past year over the five-county region in a heat map. 
We then met with our partner organization, CHP, to determine the list of stops to consider in the analysis. To start this conversation, CHP's Dental Director was presented with the following data:

- A heat map of the distribution of uninsured adults and Medicaid enrollees (Figure 1, left panel)

- A heat map of the distribution of households who earned less than $\$ 20,000$ in the past 12 months (Figure 1, right panel)

- A distance decay table (generated using the Business Analyst function in ArcMap 10.3.1) showing the number of uninsured adults and Medicaid enrollees within 5, 10, and 15 miles from each potential stop (Table A.2 in Appendix A)

- A distance decay table showing the number of households who earned less than $\$ 20,000$ in the past 12 months within 5, 10, and 15 miles from each potential stop (Table A.3 in Appendix A)

- A map showing the major road network as well as all potential stops.

Using this information, CHP determined stops that were ultimately included in this study's analysis. The places chosen as mobile stops for our baseline analysis are the following: Livingston (the hub), Wilsall, Clyde Park, Emigrant, Big Sky, and King Arthur Park. The first four locations are located in Park County, and the last two are located in Gallatin County. CHP's primary mission is to serve these two counties, so therefore our initial focus would be to examine mobile operations serving these areas. We also decided to conduct an analysis looking at places in three additional counties to see if the operation could sustainably serve the entire five-county region. The additional candidate mobile stops considered were Townsend (Broadwater County), White Sulphur Springs (Meagher County), and Big Timber (Sweet Grass County). These nine locations are shown in Figure 2.

We note that the number of stops considered in this study is smaller than the number of stops considered in other operations research-related mobile healthcare studies. For example, Lei et al. (2014) consider a stochastic programming model for mobile facility routing and scheduling that includes results for up to 15 locations, and Lei et al. (2016) consider a robust optimization model for mobile facility fleet sizing and routing that includes results for up to 30 locations. However, the two aforementioned studies focus on developing a general model and exact approaches to solve them which are computationally efficient, and not on practical limitations of their model related to healthcare operations. In another work, Hodgson et al. (1998) consider a tour-location problem that includes results for up to 50 stops. Hodgson et al. (1998) acknowledge that, "the assumption of a single tour must be relaxed in designing systems of mobile facilities" (Hodgson et al., 1998, p. 637). Doerner et al. (2007) include similar-sized instances to the aforementioned study. In their paper the authors mention, "The advantages of mobile units will have to be traded off against evident disadvantages, for example, the lack of continuous care for patients by medical personnel" (Doerner et al., 2007, p. 1093).

We consider fewer candidate stops than the aforementioned studies for practical reasons related to the lack of continuous care for patients by the medical personnel operating a single mobile clinic. Practically speaking, visiting more locations within a fixed time horizon will result in reduced frequency 
of visits to certain places, and less frequent visits to a location limits how fast patient treatment plans can be completed. To illustrate this, consider that a single mobile clinic with a four-day work week and nine candidate stops may visit certain stops only about twice per month. Although nine candidate stops may seem to be a small number, in this scenario, patients needing extensive work may be discouraged to find out that their treatment plan could take 6 to 7 months versus the 2 to 3 months associated with more frequent dentist visits, according to a mobile dentist in western Montana (J. Byington, personal communication, December 28, 2016). In sum, we believe that the timely follow-up treatment of existing patients is an important issue, and it is reflected in our set of candidate stops.

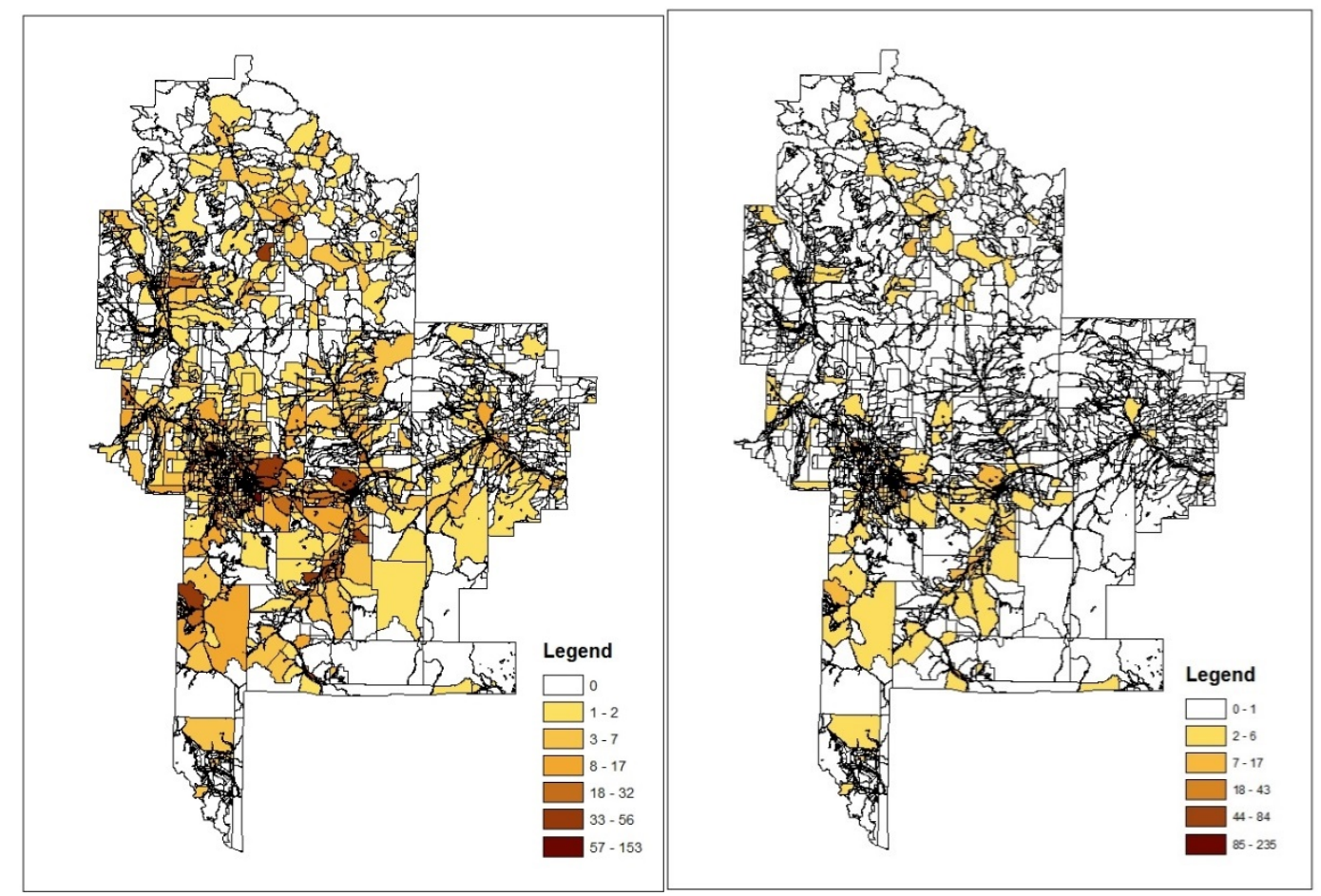

Figure 1 - Left panel: Population of the uninsured and Medicaid-enrolled population across the fivecounty region (an area covering 10,843 square miles), at Census Block level; Right panel: Number of households earning less than $\$ 20,000$ in the previous 12 months, at Census Block Level 


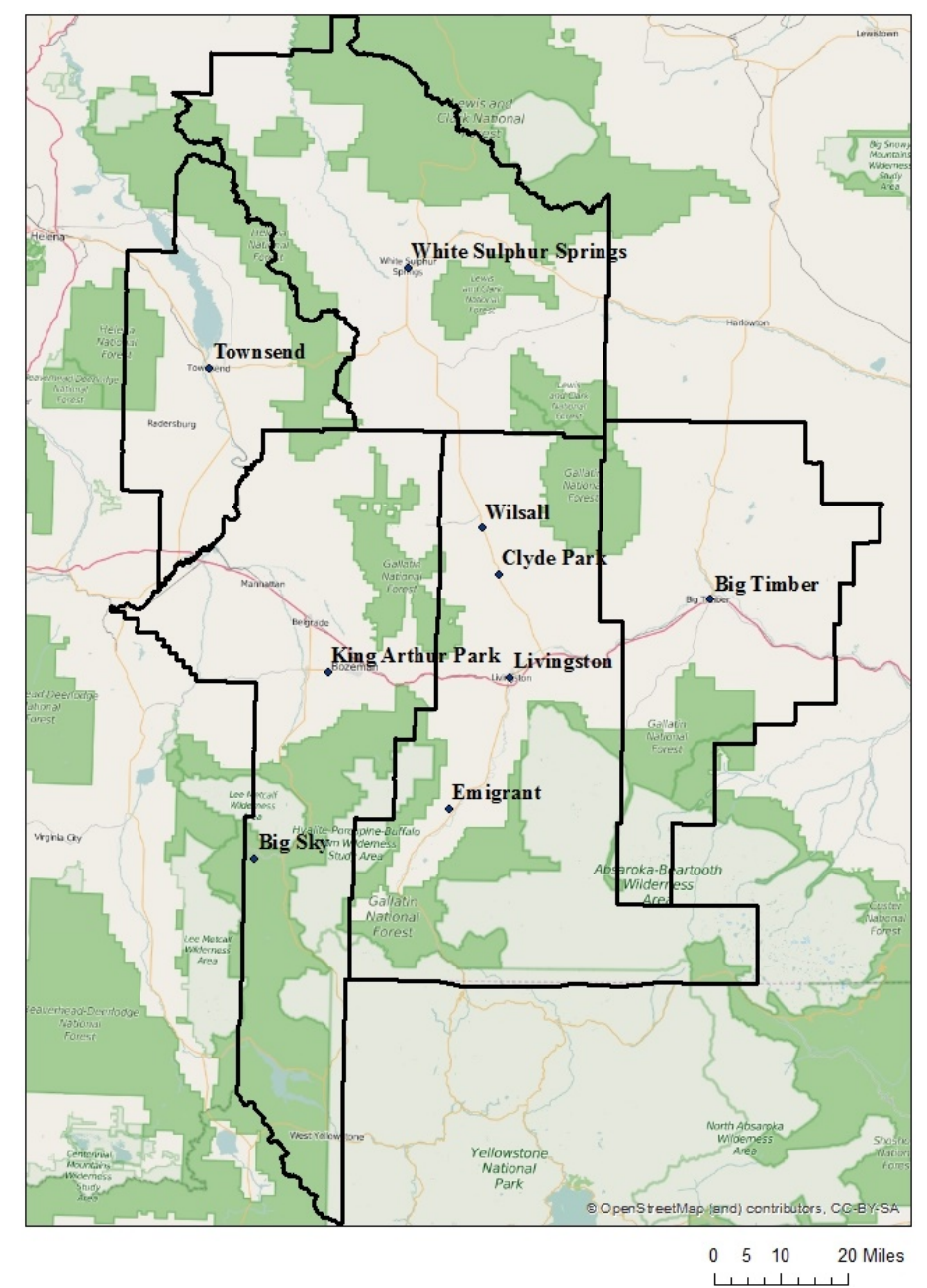

Figure 2 - Five-county region with major roadways and candidate locations

\subsection{Modeling the mobile dentistry network design problem}

In this subsection a model of MDNDP is formulated as a mixed-integer program. First, we determine the time horizon for the model. The American Dental Association (ADA) does not prescribe a one-sizefits-all interval for visits for all patients, as at-risk people may need more frequent visits than others. They do recommend professionally applied varnish at least every three to six months (ADA Center for Evidence-Based Dentistry, 2013). Based on this recommendation we consider a planning horizon of six months for a single mobile dentistry clinic in the MDNDP model.

Many mobile facility planning models have been proposed in the literature involving routing plans (e.g., Hodgson et al., 1998, Doerner et al., 2007, Lei et al., 2014, Lei et al., 2016). However, situations involving nonexempt staff (i.e., those who can accrue overtime) may require the mobile unit to return to the home location, or hub, each day, in order to manage costs. Further, setup times and breakdown times of the mobile unit may last from one to 1.5 hours, which makes multiple stops per day inefficient (M. Utley, personal communication, May 24, 2016). Seeing a high volume of patients is a key to operating a financially sustainable mobile dentistry service, especially when faced with low reimbursement rates for payers of the under-served populations (e.g., Medicaid). Therefore, our 
MDNDP model lacks routing constraints. The MDNDP model proposed can be described as an aggregate planning tool that specifies how many times to visit each location over the time horizon. The mobile vehicle can either return to the home station location at the end of each day, or, to reduce time lost to travel, the mobile operation can potentially remain overnight at a mobile service location. Notation for the MDNDP model is given below.

\section{$\underline{\text { Index sets }}$}

$i$

\section{Location $i \in I$}

Data

$a_{i} \quad$ Round trip travel time and setup/breakdown hours required to visit location $i$

$b_{i} \quad$ Total patients who can be treated per day at location $i$

c Revenue per patient

$d_{i} \quad$ Under-served population of location $i$ computed as uninsured and Medicaid-enrolled combined within a prescribed radius of the location

$e \quad$ Total fixed expense of the operation

$f \quad$ Total patients who can be treated per hour

$g \quad$ Total number of working days in horizon assuming four work days per week and two weeks of non-operation per 26-week window

$\ell \quad$ Length of workday in hours

$p_{i} \quad$ Percentage of total under-served patient population residing within a prescribed radius of location $i$ (out of total poverty patient population across all locations considered)

$\alpha \quad$ Lever to adjust sensitivity of total net revenue objective

$\beta \quad$ Lever to adjust sensitivity of service disparity (i.e., the "fairness level") across locations

$\gamma \quad$ Lever to adjust sensitivity of total patients treated

$O \quad$ Overnight stay cost per night (based on a three-night overnight trip)

$O^{\prime} \quad$ Marginal additional cost for a two-night overnight trip

$\hat{O} \quad$ Marginal additional cost for a one-night overnight trip

$Q_{i} \quad$ Additional patients treated if overnighting at location $i$, based on not having round trip travel time in the additional day

\section{Continuous variables}

$y_{i} \quad$ Total patients to be served at location $i$

$v \quad$ Total net revenue

$z \quad$ Total patients treated 
Integer variables

$x_{i} \quad$ Number of days that location $i$ is visited

$w_{i} \quad$ Total number of overnight stays at location $i$

$u_{i} \quad$ Number of three-night overnight stays at location $i$

Binary variables

$u_{i}{ }^{\prime} \quad$ Number of two-night overnight stays at location $i$

$\widehat{u_{\imath}} \quad$ Number of one-night overnight stays at location $i$

Objective Function

$$
\text { maximize } z=\sum_{i \in I} y_{i}
$$

The objective function (1) maximizes the number of patients from the under-served populations who are treated.

\section{Constraints}

$$
\sum_{i \in I} x_{i}=g
$$

Observe in constraint (2) that we visit exactly one location each day over our horizon.

$$
\begin{gathered}
y_{i}=b_{i} x_{i}+Q_{i} w_{i} \quad \forall i \\
y_{i} \leq d_{i} \quad \forall i
\end{gathered}
$$

Constraint (3) identifies the number of patients to be serviced in a location based on how many times the location has been visited and overnighted. Constraint (4) enforces that the number of patients served over the time horizon at each location is not greater than the total under-served patient population residing within a prescribed radius of that location.

$$
\begin{gathered}
v=c \sum_{i \in I} y_{i}-\sum_{i \in I}\left(O w_{i}+O^{\prime} u_{i}{ }^{\prime}+\hat{O} \widehat{u}_{\imath}\right)-e \\
v \geq \alpha \\
z \geq \gamma
\end{gathered}
$$

Constraint (5) computes the net revenue. Note that in this formulation, constraints (6) and (7) are used for sensitivity analysis, in particular for demonstrating and evaluating the tradeoff between the objective function (which maximizes the number of patients served) and net revenue (captured by parametrically solving for values of $\alpha$ ). This procedure is discussed in detail in section 4.2.

$$
\begin{array}{ll}
y_{i} \leq p_{i}(1+\beta) z & \forall i \\
y_{i} \geq p_{i}(1-\beta) z & \forall i
\end{array}
$$

Constraints (8) and (9) control the service disparity (i.e., the "fairness level") enforced by the model. When $\beta=0$, the total patients served at location $i$ is forced to equal the percentage of the total underserved patient population residing within a prescribed radius of location $i$.

$$
w_{i} \leq 0.75 * x_{i}
$$


Constraint (10) limits the number of overnights at each location to at most three nights per roundtrip, due to the assumption of a four-day workweek. As an illustration, for a location visited on eleven days, constraint (10) would ensure that no more than eight overnights occur; the maximum number of overnights corresponding to a case where two entire weeks are spent at the location (with three overnights per week), with the remaining three days corresponding to two additional overnights.

$$
3 u_{i}+2 u_{i}{ }^{\prime}+\widehat{u_{\imath}}=w_{i} \quad \forall i
$$

Constraint (11) apportions the total number of overnight stays at location $i\left(w_{i}\right)$ into a number of three-night, two-night and one-night overnight stays. The overnight stay cost is based on US GSA per diem rates (US General Services Administration, 2016), which include a lodging fee (for Montana, this rate is $\$ 91$ per night), and a meals and incidental expenses (M\&IE) rate (for Montana, this rate is $\$ 51$ per day). However, M\&IE is reduced for the first and last day of a trip, with only 75 percent of the M\&IE rate received for these days. Thus, per person, the overnight expense is $\$ 167.50, \$ 309.50$, and $\$ 451.50$ for a one-, two-, and three-night overnight stay, respectively. Observe that on a per-night basis, a three-night stay is the least-expensive option (at $\$ 150.50$ per night) and a one-night stay is the mostexpensive option. Accordingly, our formulation, which will attempt to maximize net revenue, will prefer to assign as many three-night overnight stays as possible, and would never perform more than a single two-night overnight stay or a single one-night overnight stay, which allows us to define $u_{i}{ }^{\prime}$ and $\widehat{u_{l}}$ as binary variables.

\section{The case of a five-county region in southwestern Montana}

In this section we apply the model to examine the financial viability of mobile dentistry services and its mission reach. First, the data used to parameterize the model is discussed in terms of expense data, operational data, and then revenue and demand data. Subsequently the results are presented.

\subsection{Data}

\subsubsection{Expense data}

The fixed expense considered in the model, $e$, captures the salary of employees operating the mobile service. In this case we assume that the mobile dentistry service employs one dentist, one dental hygienist, and two dental assistants. The initial analysis will be done for a service that includes three dental operatories (i.e., chairs), one used by the hygienist for cleanings, and the remaining two for the dentist to perform preventative and restorative procedures, with an assistant assigned to each of the dentist's chairs. Then assuming a 40-hour work week (four ten-hour workdays), hourly rates of $\$ 60 /$ hour (dentist), \$25/hour (dental hygienist), and \$17/hour (each dental assistant), and including an average fringe benefit rate of $23 \%$, the total expense for the six-month time horizon (assuming six months is 26 weeks) is set to $e=\$ 152,225$. The final expense is the overnight stay cost, given a four- 
person team, this expense is $\$ 670, \$ 1,238$, and $\$ 1806$ per one-, two-, and three-night overnight stay, respectively.

\subsubsection{Operational data}

As previously mentioned, in our study we assume the total number of work days, $g$, is based on a 26-week horizon. Assuming a four-day work week and two weeks of non-operation, we set $g=96$.

Round-trip travel times were found using Google Maps, and we assume an additional idle time of 60 minutes per day for setup and breakdown (30 minutes each), which was estimated by the expert stakeholders. With Livingston as the hub, the round trip travel time and setup hours for each location $i$, $a_{i}$, are the following: King Arthur Park (138 minutes), Big Sky (226 minutes), Clyde Park (108 minutes), Emigrant (112 minutes), Wilsall (124 minutes), Townsend (220 minutes), White Sulphur Springs (202 minutes), and Big Timber (126 minutes). We will limit potential overnighting to only those locations whose round-trip travel time is more than two hours from the hub at Livingston. The total number of patients treated per hour, $f$, is computed based on the staffing and the number of operatories available. In current operations at the CHP clinic, over an eight hour day with one dentist, one dental hygienist, two dental assistants, and three operatories, the dentist can treat 16 patients and the hygienist can treat 6 patients (J. Wales, personal communication, June 28, 2016). We use the same rate to parameterize our model and set $f=2.75$ patients/hour. This is used to determine the total patients who can be treated per day at location $i, b_{i}=\left\lfloor f\left(10-a_{i}\right)\right\rfloor$. The additional patients treated if overnighting at location $i, Q_{i}$, is computed similarly as $Q_{i}=\lfloor f(9)\rfloor-b_{i}$. Observe that since $b_{i}, Q_{i}, x_{i}$ and $w_{i}$ are all restricted to take only integer values, constraint (3) limits continuous variable $y_{i}$ to take only integer values.

\subsubsection{Revenue and demand data}

We set revenue per patient $c=\$ 85 /$ patient, based on the historical average observed by CHP for all patients. Assuming a fixed reimbursement rate in the model, based on historical averages, is appropriate for cases where the organization expects a similar mix of payers in the new service region. We remark that the variable expense per patient is built into the $\$ 85$ average patient reimbursement fee. Also, it must be clarified that net revenue, computed in equation (5), is defined here as total reimbursement minus the salary of employees and the overnight cost. We call this 'net revenue' in our paper for lack of existing terminology for this measure, noting that this definition does not conform to generally accepted financial accounting principles.

Demand at each location was assumed to be all adults who either have no insurance or are enrolled in Medicaid within a 15 mile radius of that location. A 15 mile radius was chosen through discussion with stakeholders, as it was agreed that it was not an excessive travel distance for patients, yet it was large enough to capture sufficient demand. Using a Business Analyst tool called 'Create Trade Area' 
within ArcMap 10.3.1, we created 15 mile radii around each location, without overlaps, where it is assumed that demand is allocated to its closest candidate stop. Then we joined the Trade Areas to the geospatial demand dataset. Table 2 shows the demand set corresponding to the nine candidate stops. This is similar to the demand from the distance decay table (Table A.2), with several slight differences and two major differences due to overlaps allowed in Table A.2. The two major differences between Table 2 and Table A. 2 are as follows: Demand at Clyde Park decreased from 1071 to 297, and demand at Wilsall decreased from 479 to 194 . Due to Wilsall's proximity to Clyde Park (7.3 miles apart), Wilsall decreased by approximately the amount of demand at Clyde Park. And demand decreased at Clyde Park due to its proximity to Livingston.

\section{Table 2.}

The number of uninsured adults and Medicaid enrollees within 5, 10, and 15 miles from each place, without overlapping

\begin{tabular}{|l|c|}
\hline & Total demand within 15 miles \\
\hline Big Sky & 412 \\
\hline Big Timber & 663 \\
\hline Clyde Park & 297 \\
\hline Emigrant & 524 \\
\hline King Arthur Park & 12323 \\
\hline Livingston & 2846 \\
\hline Townsend & 951 \\
\hline White Sulphur Springs & 503 \\
\hline Wilsall & 194 \\
\hline
\end{tabular}

\subsection{Results}

First we examine a naïve approach of treating an equal number of patients in each location, for the baseline case of six potential locations. To do this, we force an equal number of days of service at each location (over the 26-week horizon, this entails 16 days of service at each location; note that such a solution requires that we both replace the equality condition in constraint (3) with a less-than-or-equalto inequality, and also use an arbitrarily large $\beta$ value). The result is an annual net revenue of $\$ 7,500$ and 1,835 patients served (assuming no overnighting; this is the maximum-possible revenue solution). This poor solution (in terms of annual net revenue) motivates the use of optimization to find a better mix of visits per location while accruing sufficient revenue for the service to be financially viable.

Our approach to generating better solutions is as follows. By running the model over various values of $\alpha, \beta$ and $\gamma$, we can identify efficient frontiers showing the maximum number of patients in poverty (or having some other desired characteristic) that can be served at a given net revenue level $\alpha$, for a given "fairness" level $\beta$ (note that $\beta=0$ implies that the number of times each location gets visited is exactly proportional to its share of the candidate population).

The computational procedure used to generate these efficient frontiers is as follows. Denote the optimization model presented in Section 3.4 as MILP1. Define a related optimization model, MILP2, 
as having the objective to maximize $v$ (from constraint (5)), with equation (1) becoming a constraint. Let index sets $j \in J$ and $k \in K$ denote the set of potential values for $\alpha$ and $\beta$, respectively.

Algorithm 1. Generation of efficient frontiers

Step 0: Set $\beta=0$

Step 1: Loop over the elements $k \in K$

Step 1.1: Set $\alpha=-\infty$

Step 1.2: Loop over the elements $j \in J$

Step 1.2.1: Set $\gamma=0$

Step 1.2.2: Solve MILP1, recording optimal objective function value as $z^{*}$

Step 1.2.3: Set $\gamma=z^{*}$

Step 1.2.4: Solve MILP2, recording optimal objective function value as $v^{*}$

Step 1.2.5: Record $v^{*}$ and $z^{*}$ as optimal solutions for the current iterates $(j, k)$

Step 1.2.6: Set $\alpha=1.000001 * v^{*}$

Step 1.3: Set $\beta=\beta+0.01$

\subsubsection{Baseline scenario: Locations in Gallatin and Park counties}

We first examine the baseline scenario, in which six locations are considered for service, with service being offered 4 days per week and 10 hour workdays, including driving time. As the value of $\beta$ is increased, we allow the disparity (deviation between the percentage of total patients treated at each location and that location's percentage of the total target population) to increase. An increase in $\beta$ results in a relaxation of constraints (8) and (9). Accordingly, the efficient frontier shifts "up and to the right", allowing both net revenue and total patients treated to increase. For values of $\beta$ less than 0.19 , the model is infeasible, because it is impossible to obtain a solution in which each location's share of the total patients treated is exactly equal to that location's share of the total target population, due to the equality condition in constraint (3).

Observe that allowing for increased disparities across locations can greatly increase both the net revenue and the total number of treated patients. For the solution with the minimum-achievable disparities (curve corresponding to $\beta=0.19$ ), the net annual revenue that can be generated is $\$ 45,600$, with 2,067 total patients treated. By increasing the allowable disparities to $\beta=1$, the net annual revenue can be increased to a maximum of $\$ 55,100$, with 2,115 total patients treated. 


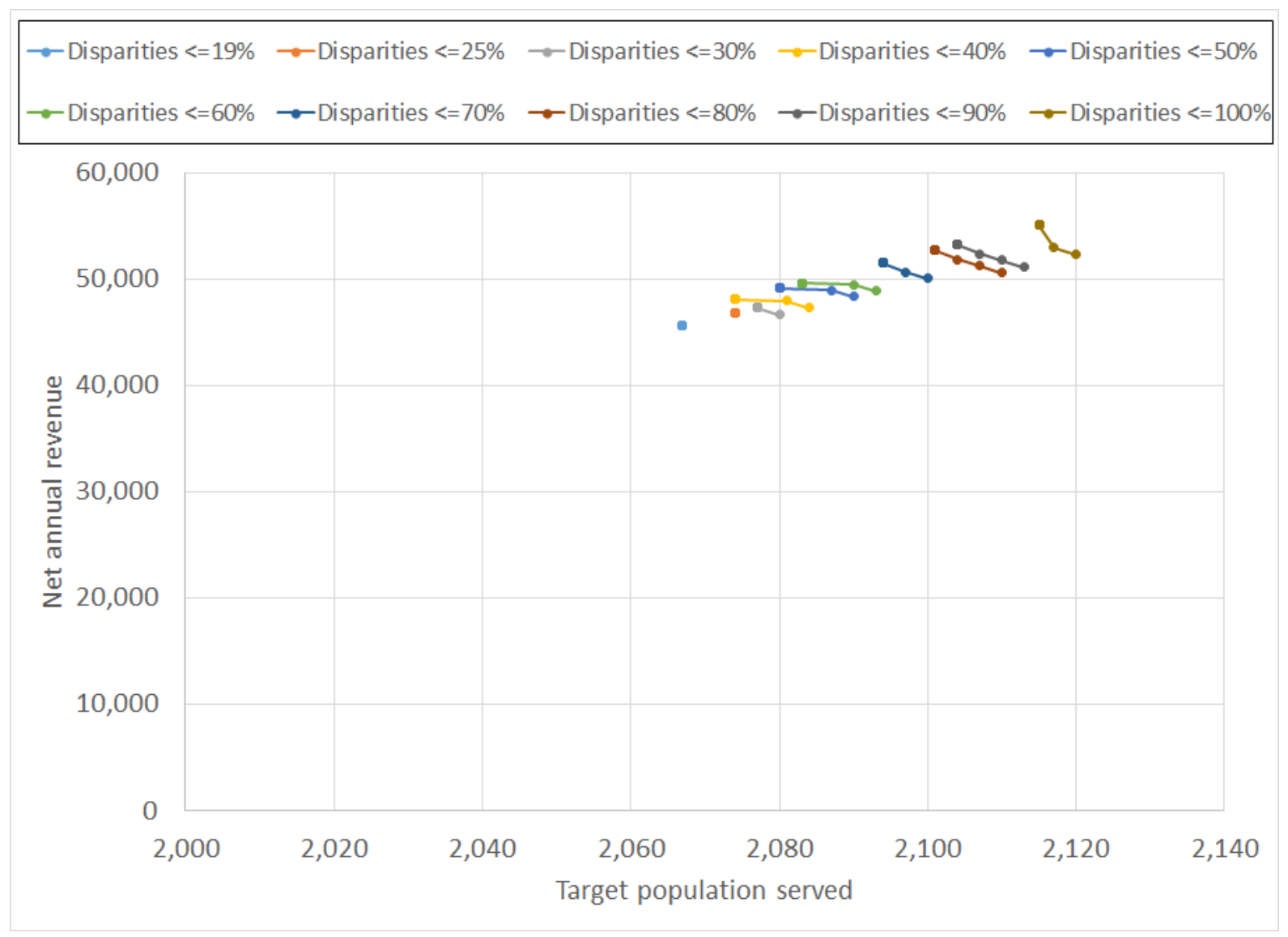

Fig. 3. Patients - revenue tradeoff curve, baseline

Allowing for increased disparities across locations can allow for increased net revenue for a constant number of patients by changing the number of patients treated at specific locations. Consider, for example, the maximum-treated-patients solution for the $\beta=0.3$ curve, this solution treats 2,080 patients with net annual revenue of $\$ 46,700$. Contrast this with the solution on the $\beta=0.5$ curve that treats the same number of patients $(2,080)$ with significantly increased net revenue of $\$ 49,200$. Figure 4 below presents the number of patients treated in each location for each of these solutions. Observe that the solution with increased revenue treats fewer patients at King Arthur Park and Big Sky (the two locations furthest from the permanent site at Livingston) and treats more patients at Emigrant and Livingston. The difference is revenue is due to the fact that the $\beta=0.3$ solution utilizes a two-night overnight stay at Big Sky per 26-week horizon (at a cost of $\$ 1,238$ ), whereas the $\beta=0.5$ solution does not utilize overnighting. 


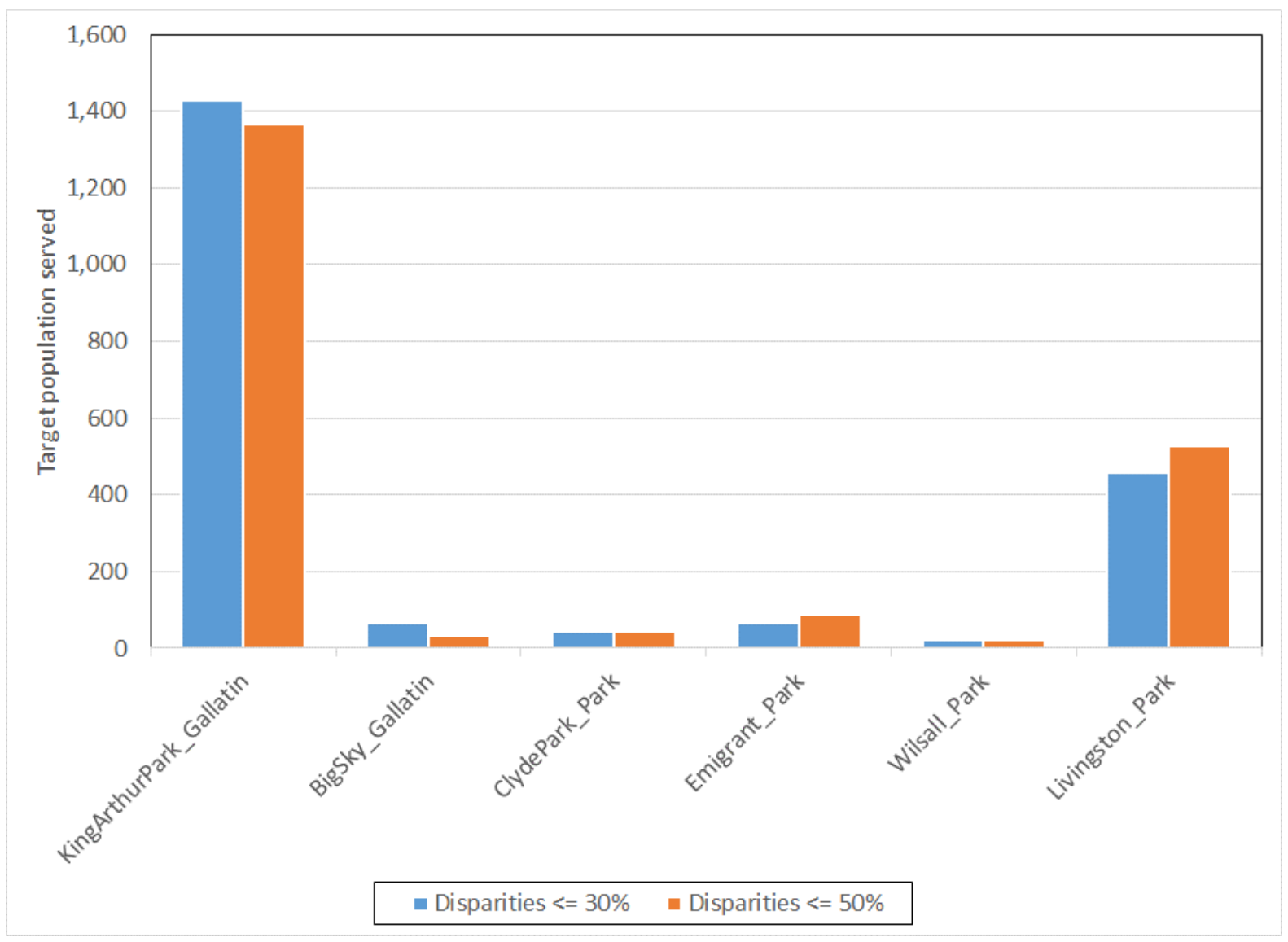

Fig. 4. Patients treated by locations, for two solutions with comparable total patients treated

\subsubsection{Expanding the set of locations - Locations in Gallatin, Park, Meagher, Broadwater, and Sweet} Grass counties

Consider next the results if the expanded set of potential locations is increased to include Townsend, White Sulphur Springs and Big Timber. Figure 5 shows the patients treated - net revenue tradeoff curve for this scenario. Observe that, for the $\beta=0.31$ curve, which is the minimum-achievable disparity for this case, the curve has shifted significantly "downward and to the left", suggesting that adding these sites will both reduce the number of patients treated and reduce net revenue, relative to the $\beta=0.3$ curve in baseline Figure 3. For all other values of $\beta$, there is little impact on the total number of patients treated if service is expanded to the three additional locations. However, across all values of $\beta \geq 0.4$, net annual revenue is decreased significantly when the set of service locations is expanded, with average decreases of 7 percent and 17 percent for the maximum-revenue and maximum-treated-patients solutions, respectively. This is because significant use of overnighting is required to obtain the necessary numbers of treated patients at Townsend and White Sulphur Springs. Figure 6 presents the $\beta=0.35$ solution with 2,079 patients treated and net revenue of $\$ 41,600$ along with the $\beta=0.7$ solution with 2,080 patients treated and net revenue of $\$ 47,800$. Here net revenue is again increased significantly by reducing the number of visits to more distant locations, with a nearly equal total number of patients treated. 


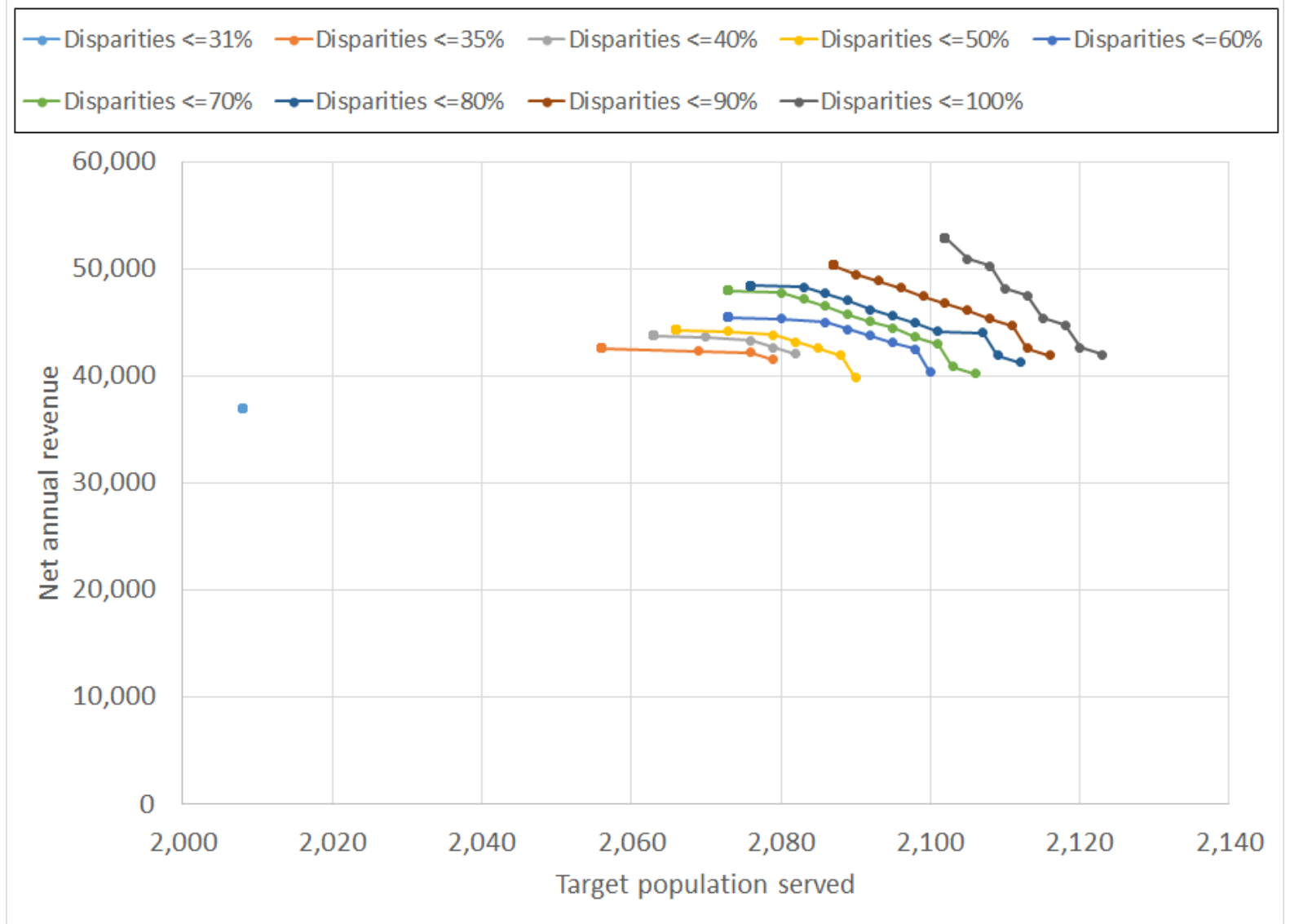

Fig. 5. Patients - revenue tradeoff curve, additional three locations 


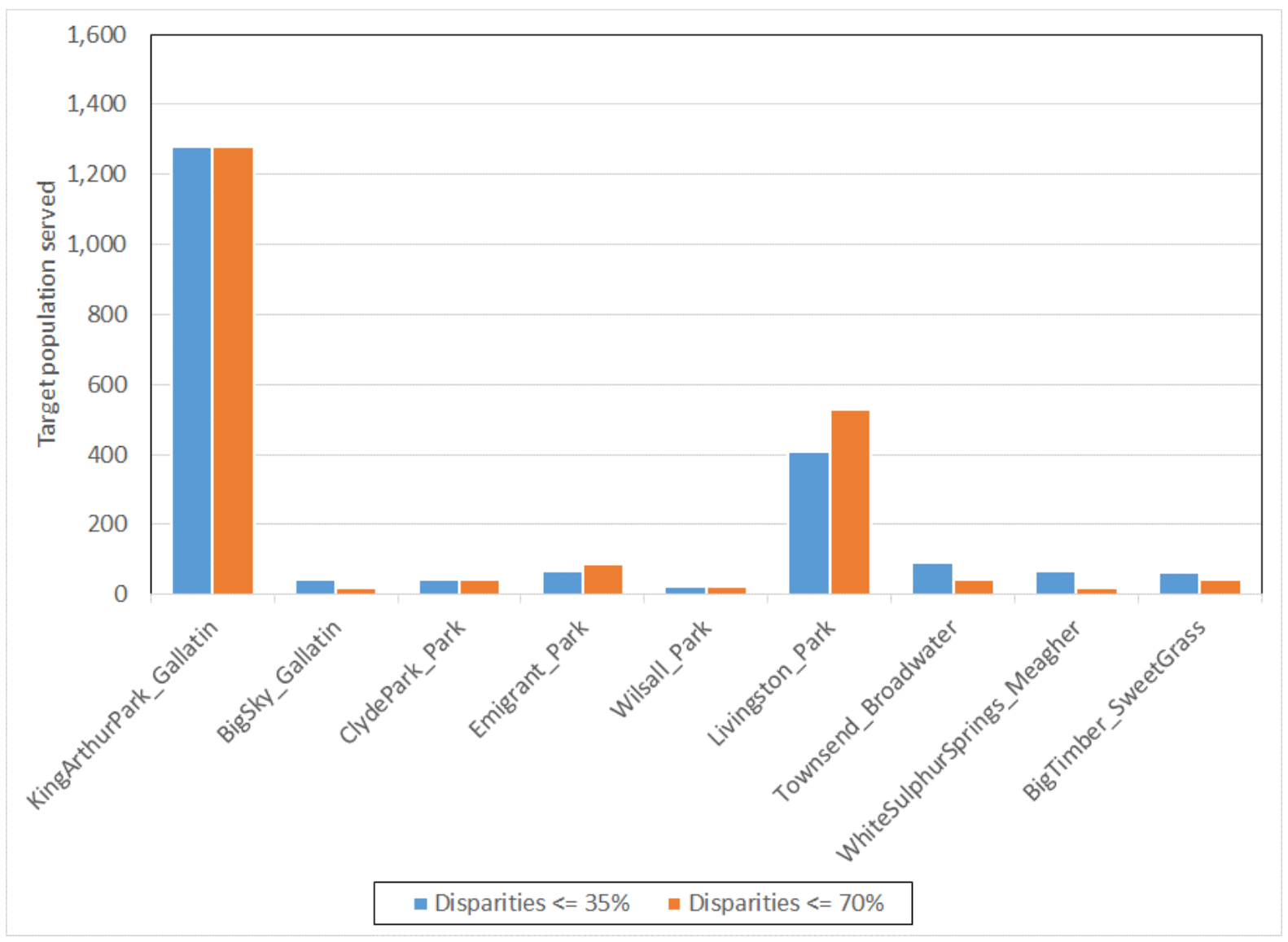

Fig. 6. Patients treated by location, for two solutions with comparable total patients treated

\subsubsection{Sensitivity analyses}

We considered a number of sensitivity analyses for these solutions. The estimate of $\$ 85$ in revenue per patient treated was based on prior experience at our partner CHP. However, consider that for individuals with commercial insurance, the average reimbursement rate to community health centers, after removing variable costs, is estimated to be $\$ 137.75$ (Apostolon, 2015). We ran the model at this increased revenue rate (assuming this provides an upper bound on the potential revenue per patient), and found that, with few exceptions, the solutions on the efficient frontiers for both cases examined previously did not change. However, the achievable net revenue increased significantly, to maximums of $\$ 278,200$ and $\$ 274,200$ for the baseline and expanded locations cases, respectively (in both instances, at $\beta=1$ ). However, this upper bound is only achievable by exclusively serving patients with commercial insurance and excluding uninsured and under-insured patients. Models were ran for two intermediate solutions evenly spread between these extremes to see the impact of milder changes to the payer mix. Average reimbursement rates of $\$ 102.58$ increased net revenue to maximums of $\$ 129,500$ and $\$ 126,800$ for the baseline and expanded locations cases, respectively (with $\beta=1$ ). Average reimbursement rates of $\$ 120.16$ increased net revenue to maximums of $\$ 203,800$ and $\$ 200,700$ for the baseline and expanded locations cases, respectively (with $\beta=1$ ).

We also performed a sensitivity analysis in which CHP provides service from a mix of fixed-site (at Livingston) and traveling operations, at the baseline $\$ 85$ revenue per patients. Assume that for one- 
half of the year, service would be provided using a standard 40 hour workweek at the Livingston hub. For these six months, there would be no travel, and thus no setup or breakdown time requirement on any day. The remaining six months would be used to provide traveling service to the other eight locations, with this service being provided in two different three-month intervals (say, from March through May, and from September through November). The disparities parameter at $\beta$ is only applied to the eight locations receiving traveling service, the number of patients treated at the fixed site in Livingston is a constant, equal to $48 *[2.75(10)\rfloor=1,296$ and is not subjected to the disparities constraint. The set of all feasible solutions under this scenario are contained on two curves, one at $\beta=$ 0.75 and the other at $\beta=1$. Figure 7 shows the patients treated - net revenue tradeoff curve for this scenario. Observe that, relative to Figure 5, both the maximum number of patients treated and the maximum net revenue can be increased under this modified work week for all achievable values of $\beta$, suggesting that such a service mix is desirable for mobile dentistry operations. However, the number of patients treated at the eight outlying sites decreases significantly under this scenario. As presented in Figure 8, the number of patients treated at the Livingston hub increases dramatically, from 528 (in the $\beta=0.7$ solution from Figure 6, corresponding to full-year mobile operations) to 1,296 under the maximum-revenue solution for semi-annual mobile operations (at $\beta=0.75$ ). However, for these same respective scenarios, the total number of patients treated across all eight other locations decreases from 1,552 in the full-year mobile operations scenario to 1,000 under the semi-annual mobile operations scenario. The corresponding increase in annual net revenue, from $\$ 47,800$ to $\$ 85,900$ demonstrates the significant positive effect of such a semi-annual mobile operations strategy on the financial sustainability of such operations, albeit at reduced service to remote locations. 


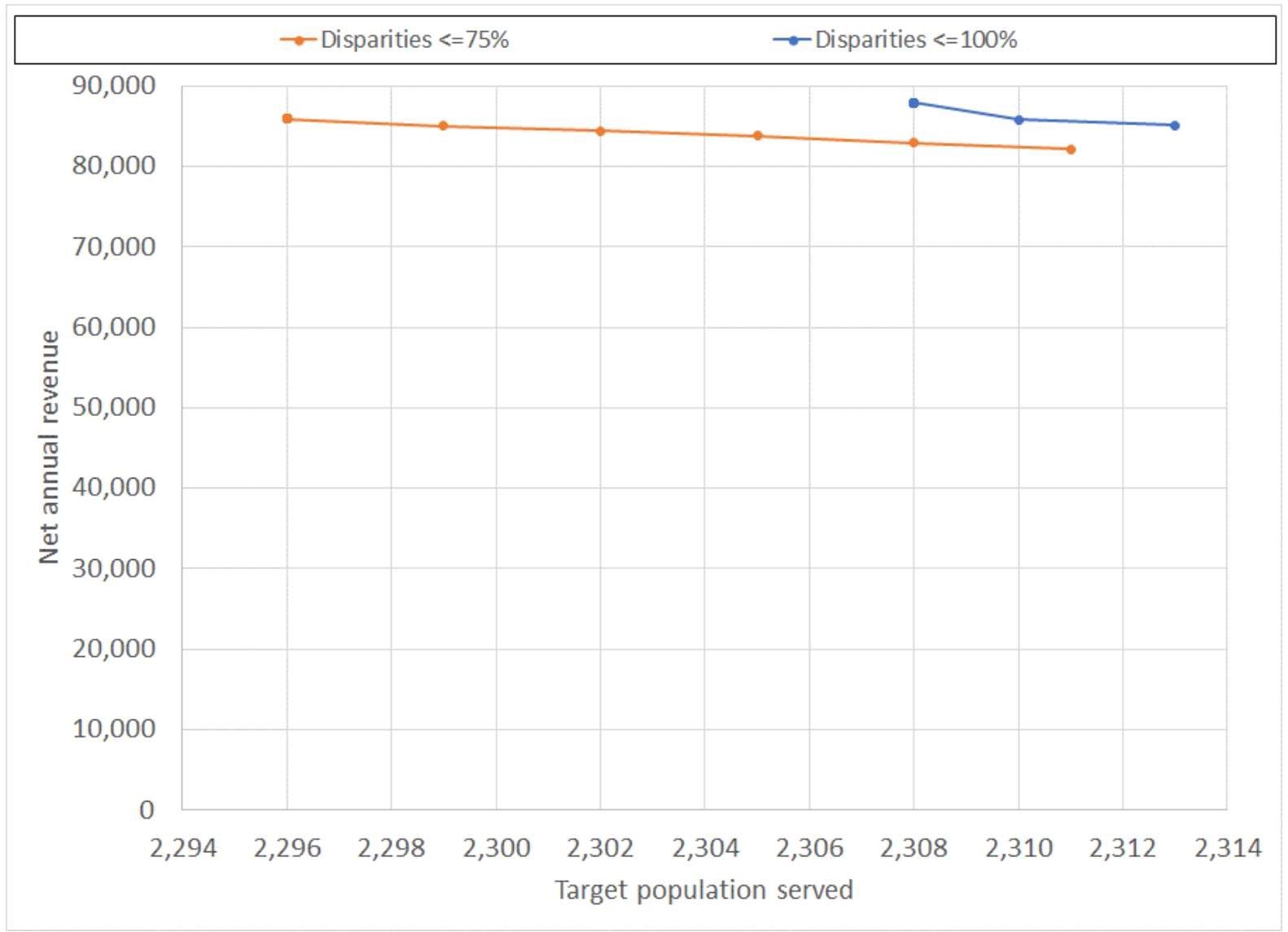

Fig. 7. Patients - revenue tradeoff curve, traveling semi-annually 


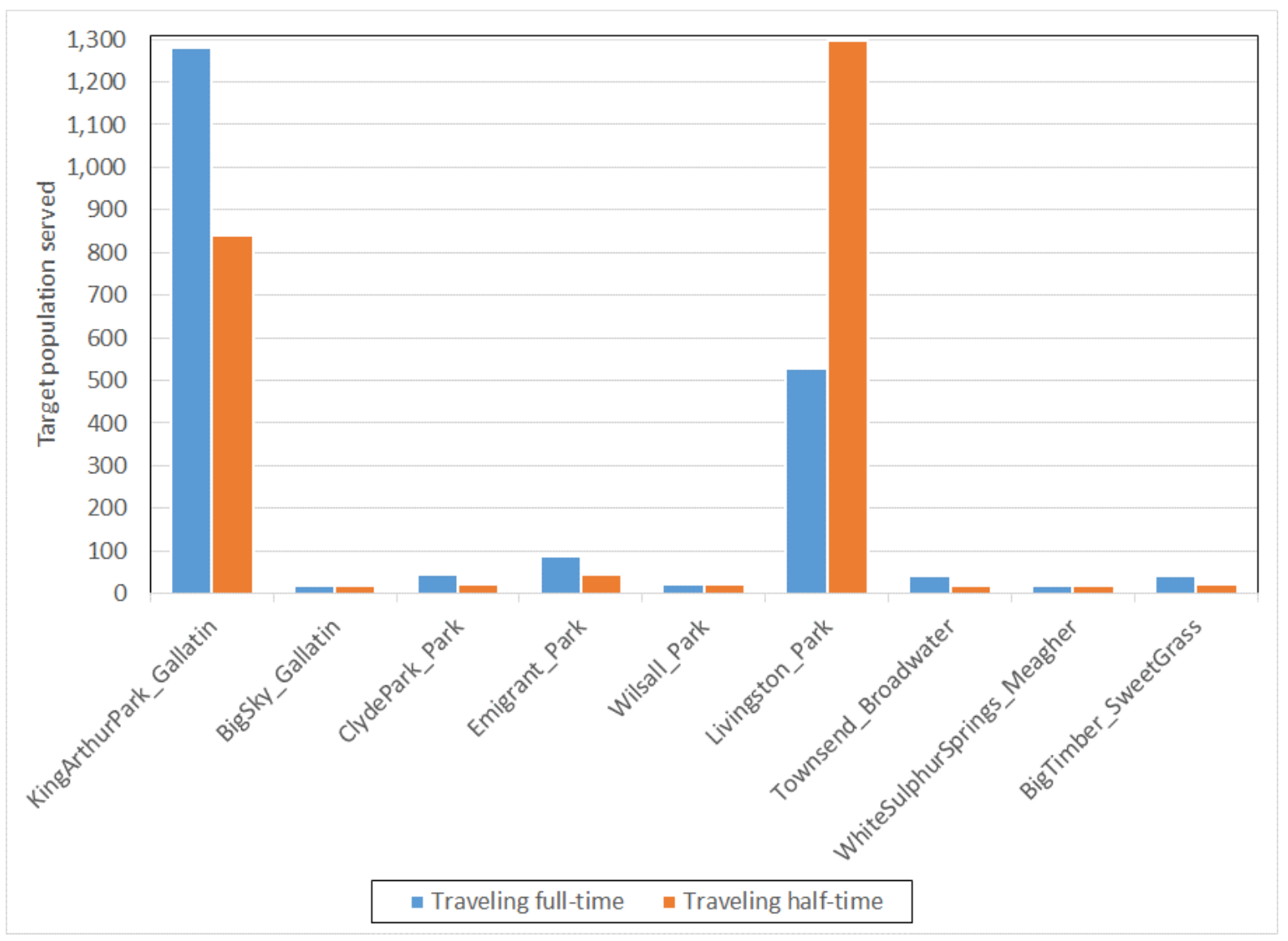

Fig. 8. Patients treated by location, traveling full-time versus traveling semi-annually

\section{Discussion}

Our results clearly demonstrate that operating a mobile dentistry service in a rural region is financially challenging. The annual net revenue modeled in our experiments, which captures employee costs, is an essential piece of determining whether a service can maintain financial sustainability. Our results found net revenue to range from around $\$ 50,000$, when service is provided on a traveling basis to all locations, to approximately $\$ 85,000$, when service is provided for six months per year at the fixed site in Livingston and for the remaining six months on a traveling basis to the other locations. The financial challenge is due to costs left out of our analysis which may be larger than the net revenue generated. Specifically, several large costs were left out of the analysis, including start-up costs of purchasing the equipment and annual fixed costs. Even when assuming that start-up costs for the vehicle purchase are covered by a charitable donation (e.g., the situation in Granite County, Montana described by Briggeman (2013)), it will still be challenging for net revenue to cover the annual costs associated with the service. Unless the organization has administrative slack, an additional administrative employee and a laptop with a software management system may be needed to perform duties such as patient scheduling, collect bills, and recordkeeping (DentaQuest, n.d.). Other costs include malpractice insurance, insurance and maintenance for the vehicle, phone service, office supplies, disposable dental 
supplies and instruments. For instance, there can be unanticipated costs associated with "fuel, repairs, maintenance, security, insurance and storage" (Moulavi, Bushy, Peterson \& Stullenbarger, 1999).

Two characteristics of our results were very consistent: First, we notice throughout the results that reducing the number of visits to locations that are distant from the hub has a positive effect on revenue. This is because overnight stays are required to provide service to a large number of patients at these locations, incurring additional costs.

The second characteristic of our results that was very consistent was the high rate of visits in Livingston. This is simply because the model prefers to serve larger populations closer to the hub (Livingston) to reduce travel time. A supplemental analysis was conducted to address this as followed. We solved the entire set of efficient frontiers while enforcing constraints that at least 100 patients in each of the nine locations are served. In order to generate a feasible solution, we must set $\beta=361 \%$. This solution generates an annual net revenue of $\$ 58,500$.

Table 3.

Solution of MILP2 enforcing a constraint that at least 100 patients in each of the nine locations are served

\begin{tabular}{lcc}
\hline \multicolumn{1}{c}{ Location } & Number of patients served & Number of visits per six months \\
\hline King Arthur Park & 105 & 5 \\
Big Sky & 106 & 5 (3 overnights $)$ \\
Clyde Park & 110 & 5 \\
Emigrant & 110 & 5 \\
Wilsall & 105 & 5 \\
Livingston & 1344 & 56 \\
Townsend & 106 & 5 (3 overnights $)$ \\
White Sulphur Springs & 108 & $5(3$ overnights $)$ \\
Big Timber & 105 & 5 \\
\hline
\end{tabular}

The high rate of visits in Livingston may be practical in southwestern Montana. The region can experience cold, snowy winters, suggesting that it would be preferable to schedule service at the hub (in the winter) to reduce the impact of travel issues. These considerations provide further support to the strategy that spends one-half of the year providing service out of the Livingston hub.

With low reimbursement rates for serving an under-served population, volume may be a key to financial sustainability. In our study we assume the vehicle has three operatories serving a total of 2.75 patients/hour. Patient throughput would decrease significantly if only two chairs were available, which could dramatically effect net annual revenue and annual patients treated. Therefore, reduced spending for lower vehicle capacity would be a risky decision. If a more complete identification of other costs were completed, and found to exceed the net revenue estimated by this analysis, this shortfall could be identified (and, potentially presented to charitable foundation funding partners) as an annual charitable donations requirement needed to sustain the operation. 
One approach to achieve financial sustainability is to serve patients who may generate higher revenue. De Véricourt and Lobo (2009) show that nonprofits can serve more mission-related customers with increased capacity, where the capacity can be obtained by serving more revenue-generating patients. It seems like geographically isolated rural populations who are insured (and non-Medicaid, non-Medicare) would value and could monetarily support mobile services. Therefore, our sensitivity analysis conducted on reimbursement rates in the previous section can be viewed as experimenting with "expanding the boundaries" of the organization from serving under-insured people to serving rural people in general. In particular, we solve our models using reimbursement rates ranging from status quo (revenue per patient $=\$ 85 /$ patient) to a reimbursement rate representing a payer mix of $100 \%$ commercial insurance (revenue per patient $=\$ 137.75 /$ patient) and several rates in between. We find that a substantial increase in net revenue can be achieved by increases reimbursement rates. We also note that, instead of changing the payer mix significantly (thus reducing service to the under-served population), it may be appropriate to bridge the gap between the status quo reimbursement rate and a target reimbursement rate for sustainability through welfare subsidies.

The approach described in this paper can be generalized to assist in the planning for any mobile health service. Positive results and impact can be achieved when working with industry partners (e.g. CHP) and engaging with other parties, public and private, who are both knowledgeable about general operational issues for the service (so that models mimic reality as much as possible), who have information about specific regional constraints (e.g. our discussions with several mobile dentists in Montana), and who have a vested interest in the betterment of the health of local people (e.g. DPHHS).

The model and routing recommendations posed in this paper helped to quantify the need for mobile dentistry in the five-county region studied using GIS at a granular level for the first time. Further, the model solution developed insights about the resources required to operate a prospective mobile dentistry service. To the best of our knowledge, this is the first study using an optimization-based approach (more specifically, a CBOR approach) to study the financial sustainability of mobile dentistry services. For real-world applications, understanding this issue is paramount for determining whether the proposed operation is practical. Generally, given the assumptions we made, it seemed clear to CHP that mobile dentistry services would be difficult to sustain without a significant amount of additional subsidies.

As mentioned earlier, previous research on mobile healthcare systems coming from the operations research community did not consider financial sustainability of the overall system. This is an important oversight because sometimes technically-correct and well-intentioned decision models may have challenges in generating recommendations that are financially, operationally, and socially feasible. In order for operations research models to be successfully applied, especially, but not limited to, not-forprofit and public sector applications, substantial engagement with stakeholders throughout the modeling process (e.g., the CBOR approach undertook in this paper) is necessary.

\section{Limitations and Future Research Directions}




\subsection{Limitations}

A limitation with respect to the data used in this study is related to the unavailability of datasets on dental insurance coverage rates in the US over small geographic areas. Consequently, we used available data on health insurance coverage rates, which is widely known to be a conservative approximation of the number of people without dental insurance. These data were appropriate for our study since they represented the upper bound for population served at each location; these upper bounds were never tight, with potential demand always exceeding supply. However, we acknowledge that our data may underestimate actual demand, and we would not claim that our model serves all demand because the under-served population is very large in the region.

Another limitation of this study is with respect to the process used to choose candidate locations. Places, as defined by the US Census Bureau, in the five-county region were chosen as candidate locations since these settled concentrations of populations may have enough demand to fill the mobile clinics schedule. Indeed, with the 15 mile distance constraint, the nine stops cover 18,713 of the 21,314 people in our demand set (87.8\%). However, a limitation of this approach to choose candidate locations is that the most sparsely populated areas may be effectively ignored. Specifically, in terms of the physical area covered, the set of stops covers $5,068 \mathrm{mi}^{2}$ out of the total $10,941 \mathrm{mi}^{2}$ that comprises the five-county region, or $46.32 \%$ of the five-county region. This is because several of the radii overlap, and a few of the stops are close to the boundary of the region. Had we placed the 9 stops without any overlapping and the service circles completely inside of the five-county region, the maximum coverage would be $\left(706 \mathrm{mi}^{2}\right)^{* 9}=6,354 \mathrm{mi}^{2}$, or $58.08 \%$ of the five-county region. So, in terms of maximizing service coverage, the set of candidate stops may be suboptimal. At first glance, using this approach may appear counter to the objectives of the current study (that is, the objective of increasing dental service access in rural areas). It can alternatively be reasoned that assessing the financial sustainability of a mobile dentistry model is the primary objective of the study. Once a sustainable business model is developed and successfully implemented, word may spread about the service, and given sufficient resources, additional locations can be added in those more sparsely populated regions. Our study addresses extending services to additional locations by first conducting an analysis consisting of stops located in only two counties (Gallatin and Park), and then conducting another analysis consisting of stops located in all five counties in CHP's mission area, and finds that expanding to five counties decreases annual net revenue.

Further, we note that our study assumed that the mobile operation had three operatories. However, there are different styles of mobile units that can offer three operatories, including vans and semitrailers. It is likely that some of the staff will travel with the mobile unit and others will travel separately which may allow for other efficiencies to be had, such as the unit remaining set up on site while some staff go home to overnight, a situation where set-up/breakdown time is eliminated as well as per diem 
costs. However, here may also be additional costs with operating a semi-trailer such as needing to hire a trained driver. These additional costs can be added into the model in the future.

\subsection{Future research directions}

There are several future research directions that would be of interest. While the models described in this paper did not produce highly profitable operations, including other services to increase revenue may be useful (e.g., serving elder care facilities, pediatric patients, or American Indian reservations). Another approach that may improve the financial sustainability of this operation would be to serve rural people more broadly (i.e., not only uninsured and Medicaid patients). It is possible that the rural population may value mobile services and could support them through payments provided by private insurance. Therefore, an extension of the model could determine an optimal mix of patients who are characterized by the procedure or payment method (e.g., sliding-scale fee for uninsured, Medicaid, and private insurances).

Future research should also consider incorporating information on patient no-shows into their models. No-shows make a significant impact on performance measures such as server utilization and revenue generation. In particular, the service rate assumed in this paper of 2.75 patients per hour would decrease with no-shows. A mobile dentist in western Montana reported that there was an issue with noshows when the service in Seeley was first started, but few once it was established (J. Byington, personal communication, June 1, 2016). This dentist also reported that new patients are the biggest no-show risk, so they maintain a list of patients who they can call for last-minute appointments in case of a no-show. The service rate data assumed in the model was conservative to take into account issues such as patient no-shows. Many industries use overbooking policies to mitigate the effect of no-shows, however it is unclear what sort of policy would be optimal for mobile dentistry. The prospect of turning away patients or working overtime are both unattractive options. Perhaps procedures that can be performed simultaneously across operatories would be good candidates for overbooked appointments, and that could reduce the impact on overtime. An empirical study on no-show rates for patients with different characteristics in the context of mobile dentistry could also be of benefit.

\section{Acknowledgements}

We are grateful for the rich discussions, from the very beginning of this project, with Lander Cooney and Dr. Joanna Wales, DDS, from CHP. Also, we appreciate the time spent with the following individuals who provided information about mobile dentistry services and the dental landscape in Montana: Tonette Hollingsworth, Oral Health Program Coordinator, Montana Department of Health and Human Services; Dr. Joe Byington, DDS, Partnership Health Center; Melissa Utley, RDH LAP, Dental Hygienist, Program Manager, St. Vincent's Healthcare, Ronald McDonald Care Mobile. Finally we thank Dr. Maggie Thorsen (Assistant Professor of Sociology, Montana State University) whose comments and suggestions strengthened the paper. 
This research did not receive any specific grant from funding agencies in the public, commercial, or not-for-profit sectors.

\section{Appendix A}

Table A.1 presents the places, as identified by the US Census Bureau in the 2010 decennial census, for the five-county region. Tables A.2 and A.3 present distance decay data for several attributes of the population in the same region.

Table A.1.

List of all places in target counties identified by 2010 census (US Census Bureau, 2012) Legal/statistical area

\begin{tabular}{|c|c|c|c|}
\hline County & Place & description & Population \\
\hline \multirow[t]{20}{*}{ Gallatin } & Amsterdam & census-designated place & 180 \\
\hline & Belgrade & city & 7,389 \\
\hline & Bozeman & city & 37,280 \\
\hline & Bridger & census-designated place & 30 \\
\hline & Churchill & census-designated place & 902 \\
\hline & Four Corners & census-designated place & 3,146 \\
\hline & $\begin{array}{l}\text { Gallatin Gateway } \\
\text { Gallatin River }\end{array}$ & census-designated place & 856 \\
\hline & Ranch & census-designated place & 69 \\
\hline & Hebgen Lake & & \\
\hline & Estates & census-designated place & 70 \\
\hline & King Arthur Park & census-designated place & 738 \\
\hline & Logan & census-designated place & 99 \\
\hline & Manhattan & town & 1,520 \\
\hline & Ponderosa Pines & census-designated place & 336 \\
\hline & Sedan & census-designated place & 99 \\
\hline & Springhill & census-designated place & 130 \\
\hline & Three Forks & city & 1,869 \\
\hline & West Yellowstone & town & 1,271 \\
\hline & Willow Creek & census-designated place & 210 \\
\hline & Big Sky & census-designated place & 2,308 \\
\hline \multirow[t]{12}{*}{ Park } & Clyde Park & town & 288 \\
\hline & Cooke City & census-designated place & 75 \\
\hline & Corwin Springs & census-designated place & 109 \\
\hline & Emigrant & census-designated place & 488 \\
\hline & Gardiner & census-designated place & 875 \\
\hline & South Glastonbury & census-designated place & 284 \\
\hline & Jardine & census-designated place & 57 \\
\hline & Livingston & city & 7,044 \\
\hline & Pray & census-designated place & 681 \\
\hline & Silver Gate & census-designated place & 20 \\
\hline & Springdale & census-designated place & 42 \\
\hline & Wilsall & census-designated place & 178 \\
\hline
\end{tabular}




\begin{tabular}{llll} 
& Wineglass & census-designated place & 256 \\
\hline Broadwater & Radersburg & census-designated place & 66 \\
& Spokane Creek & census-designated place & 355 \\
& The Silos & census-designated place & 506 \\
& Toston & census-designated place & 27 \\
& Townsend & city & 1,878 \\
& Wheatland & census-designated place & 568 \\
& Winston & census-designated place & 147 \\
\hline \multirow{2}{*}{ Meagher } & Martinsdale & census-designated place & 64 \\
& White Sulphur & & \\
& Springs & city & 939 \\
\hline \multirow{2}{*}{ Sweet Grass } & Big Timber & city & 1,641 \\
& Greycliff & census-designated place & 112 \\
\hline
\end{tabular}

Table A.2.

The number of uninsured adults and Medicaid enrollees within 5, 10, and 15 miles from each place

\begin{tabular}{|c|c|c|c|c|c|}
\hline County & Place & $0-5$ miles & 5-10 miles & $10-15$ miles & Total within $15 \mathrm{mi}$. \\
\hline \multirow[t]{19}{*}{ Gallatin } & Amsterdam & 392 & 4255 & 6367 & 11014 \\
\hline & Belgrade & 3661 & 5967 & 3099 & 12727 \\
\hline & Bozeman & 6326 & 2641 & 3228 & 12195 \\
\hline & Bridger & 30 & 701 & 8694 & 9425 \\
\hline & Churchill & 820 & 4192 & 6707 & 11719 \\
\hline & Four Corners & 2040 & 9630 & 967 & 12637 \\
\hline & $\begin{array}{l}\text { Gallatin Gateway } \\
\text { Gallatin River }\end{array}$ & 906 & 6714 & 4416 & 12036 \\
\hline & $\begin{array}{l}\text { Ranch } \\
\text { Hebgen Lake }\end{array}$ & 518 & 967 & 3551 & 5036 \\
\hline & Estates & 36 & 515 & 1 & 552 \\
\hline & King Arthur Park & 6500 & 4814 & 1035 & 12349 \\
\hline & Logan & 365 & 988 & 3453 & 4806 \\
\hline & Manhattan & 556 & 3261 & 2045 & 5862 \\
\hline & Ponderosa Pines & 53 & 58 & 1345 & 1456 \\
\hline & Sedan & 13 & 85 & 401 & 499 \\
\hline & Springhill & 121 & 3768 & 7062 & 10951 \\
\hline & Three Forks & 664 & 156 & 605 & 1425 \\
\hline & West Yellowstone & 505 & 39 & 5 & 549 \\
\hline & Willow Creek & 56 & 673 & 141 & 870 \\
\hline & Big Sky & 303 & 45 & 64 & 412 \\
\hline \multirow[t]{9}{*}{ Park } & Clyde Park & 223 & 230 & 618 & 1071 \\
\hline & Cooke City & 12 & 2 & 0 & 14 \\
\hline & Corwin Springs & 63 & 205 & 99 & 367 \\
\hline & Emigrant & 307 & 163 & 155 & 625 \\
\hline & Gardiner & 207 & 46 & 8 & 261 \\
\hline & South Glastonbury & 140 & 234 & 159 & 533 \\
\hline & Jardine & 139 & 70 & 44 & 253 \\
\hline & Livingston & 2412 & 269 & 316 & 2997 \\
\hline & Pray & 271 & 285 & 131 & 687 \\
\hline
\end{tabular}




\begin{tabular}{llllll} 
& Silver Gate & 14 & 0 & 0 & 14 \\
& Springdale & 22 & 64 & 505 & 591 \\
& Wilsall & 118 & 255 & 106 & 479 \\
& Wineglass & 2237 & 479 & 368 & 3084 \\
\hline Broadwater & Radersburg & 30 & 170 & 590 & 790 \\
& Spokane Creek & 82 & 47 & 134 & 263 \\
& The Silos & 143 & 608 & 204 & 955 \\
& Toston & 69 & 134 & 621 & 824 \\
& Townsend & 572 & 216 & 163 & 951 \\
& Wheatland & 614 & 186 & 550 & 1350 \\
& Winston & 86 & 180 & 533 & 799 \\
\hline Meagher & Martinsdale & 37 & 11 & 16 & 64 \\
& White Sulphur & & & & \\
& Springs & 392 & 85 & 26 & 503 \\
\hline \multirow{5}{*}{ Sweet Grass } & Big Timber & 527 & 78 & 58 & 663 \\
& Greycliff & 85 & 517 & 55 & 657 \\
\hline
\end{tabular}

Table A.3.

The number of households who earned less than $\$ 20,000$ in the past 12 months within 5, 10, and 15 miles from each place

\begin{tabular}{llllll} 
County & Place & $0-5$ miles & $5-10$ miles & $10-15$ miles & Total within 15 mi. \\
\hline Gallatin & Amsterdam & 55 & 1015 & 3501 & 4571 \\
& Belgrade & 867 & 2862 & 1580 & 5309 \\
& Bozeman & 3840 & 586 & 808 & 5234 \\
Bridger & 6 & 105 & 4371 & 4482 \\
& Churchill & 202 & 958 & 3892 & 5052 \\
& Four Corners & 822 & 4327 & 156 & 5305 \\
Gallatin Gateway & 277 & 3934 & 1012 & 5223 \\
Gallatin River & & & & \\
& Ranch & 84 & 190 & 826 & 1100 \\
& Hebgen Lake & & & & \\
& Estates & 17 & 122 & 0 & 139 \\
King Arthur Park & 3922 & 1169 & 161 & 5252 \\
& Logan & 60 & 207 & 812 & 1079 \\
& Manhattan & 94 & 771 & 426 & 1291 \\
& Ponderosa Pines & 15 & 9 & 256 & 280 \\
& Sedan & 2 & 17 & 53 & 72 \\
Springhill & 20 & 863 & 3859 & 4742 \\
& Three Forks & 149 & 37 & 114 & 300 \\
West Yellowstone & 118 & 19 & 2 & 139 \\
& Willow Creek & 14 & 151 & 34 & 199 \\
Big Sky & 62 & 19 & 29 & 110 \\
\hline Park & Clyde Park & 26 & 32 & 146 & 204 \\
Cooke City & 1 & 0 & 0 & 1 \\
Corwin Springs & 20 & 78 & 21 & 119 \\
Emigrant & 70 & 39 & 40 & 149 \\
Gardiner & 79 & 14 & 2 & 95 \\
& & & & &
\end{tabular}




\begin{tabular}{llllll} 
& South Glastonbury & 31 & 51 & 46 & 128 \\
& Jardine & 52 & 28 & 13 & 93 \\
& Livingston & 821 & 53 & 65 & 939 \\
& Pray & 64 & 69 & 47 & 180 \\
& Silver Gate & 1 & 0 & 0 & 1 \\
& Springdale & 7 & 10 & 143 & 160 \\
& Wilsall & 20 & 30 & 15 & 65 \\
& Wineglass & 778 & 119 & 67 & 964 \\
\hline \multirow{6}{*}{ Broadwater } & Radersburg & 6 & 31 & 233 & 270 \\
& Spokane Creek & 19 & 10 & 34 & 63 \\
& The Silos & 39 & 230 & 34 & 303 \\
& Toston & 6 & 19 & 242 & 267 \\
& Townsend & 229 & 41 & 20 & 290 \\
& Wheatland & 137 & 46 & 95 & 278 \\
& Winston & 21 & 44 & 218 & 283 \\
\hline Meagher & Martinsdale & 6 & 2 & 2 & 10 \\
& White Sulphur & & & & \\
& Springs & 72 & 16 & 5 & 93 \\
\hline Sweet Grass & Big Timber & 177 & 16 & 14 & 207 \\
& Greycliff & 19 & 173 & 12 & 204 \\
\hline
\end{tabular}




\section{References}

ADA Center for Evidence-Based Dentistry (2013). Topical fluoride for caries prevention http://ebd.ada.org/ /media/EBD/Files/Topical fluoride for_caries prevention_2013 update.ashx Accessed 1.5.2016.

Alliance for Health Reform. (2012). Oral health and the Affordable Care Act: Only part way to the finish line. http://www.allhealth.org/publications/Oral_Health_Issue_Brief_116.pdf Accessed 28.7.2016.

Apostolon, D. (2015). Dental program performance [Presentation slides]. Retrieved from http://www.nnoha.org/nnoha-content/uploads/2015/12/YOHP-Session-6-Dental-ProgramPerformance.pdf Accessed 20.1.2017.

Briggeman, K. (2013, April 9). Retrofitted room at Philipsburg medical center serves as dental clinic. Missoulian, http://www.missoulian.com

DentaQuest Institute. (n.d.) Staffing by Design. https://www.dentaquestinstitute.org/system/files/Staffing\%20Basics.pdf Accessed 27.7.2016.

Davis, M. M., Hilton, T. J., Benson, S., Schott, J., Howard, A., McGinnis, P., \& Fagnan, L. (2010). Unmet dental needs in rural primary care: a clinic-, community-, and practice-based research network collaborative. The Journal of the American Board of Family Medicine, 23(4), 514-522.

De Véricourt, F., \& Lobo, M. S. (2009). Resource and revenue management in nonprofit operations. Operations Research, 57(5), 1114-1128.

Doerner, K., Focke, A., \& Gutjahr, W. J. (2007). Multicriteria tour planning for mobile healthcare facilities in a developing country. European Journal of Operational Research, 179(3), 1078-1096.

Guay, A. H. (2005). The oral health status of nursing home residents: what do we need to know? Journal of Dental Education, 69(9), 1015-1017.

HealthCare.gov (n.d.) Federal Poverty Level. https://www.healthcare.gov/glossary/federal-povertylevel-FPL/ Accessed 27.7.2016.

Hindle, G. A., \& Vigden, R. (2017) Developing a business analytics methodology: a case study in the foodbank sector. European Journal of Operational Research, under review.

Hodgson, M. J., Laporte, G., \& Semet, F. (1998). A covering tour model for planning mobile health care facilities in Suhum District, Ghana. Journal of Regional Science, 38(4), 621-63.

Hollister, M. C., \& Weintraub, J. A. (1993). The association of oral status with systemic health, quality of life, and economic productivity. Journal of Dental Education, 57(12), 901-912.

Johnson, M. P. (2006). Decision models for the location of community corrections centers. Environment and Planning B: Planning and Design, 33(3), 393-412.

Johnson, M. p., \& Smilowitz, K. (2007). Community-based operations research. In Tutorials in Operations Research 2007. Klastorin T (ed.). INFORMS, Hanover, MD. 
Johnson, M. P., Midgley, G., \& Chichirau, G. (2017). Emerging trends and new frontiers in community operational research. European Journal of Operational Research, under review.

Johnson, M. P., \& Smilowitz, K. (2012). Tutorials in Operations Research 2007: "Community-Based Operations Research.” In M. P. Johnson (Ed.), Community-Based Operations Research: Decision Modeling for Local Impact and Diverse Populations (pp. 37-65). New York: Springer.

Kimminau, K., \& Wellever, A. (2011). Mapping the Rural Kansas Dental Workforce. Implications for Population Oral Health. Topeka, KS: Bureau of Oral Health, Kansas Department of Health and Environment.

Kiyak, H. A., \& Reichmuth, M. (2005). Barriers to and enablers of older adults' use of dental services. Journal of Dental Education, 69(9), 975-986.

Lei, C., Lin, W. H., \& Miao, L. (2014). A multicut L-shaped based algorithm to solve a stochastic programming model for the mobile facility routing and scheduling problem. European Journal of Operational Research, 238(3), 699-710.

Lei, C., Lin, W. H., \& Miao, L. (2016). A two-stage robust optimization approach for the mobile facility fleet sizing and routing problem under uncertainty. Computers \& Operations Research, 67, 75-89.

Medicaid.gov. (n.d.) Dental Care. https://www.medicaid.gov/medicaid/benefits/dental/index.html Accessed 23.12.2016.

Midgley, G., Johnson, M. P., \& Chicirau, G. (2017). What is community operational research? European Journal of Operational Research, under review.

Montana Department of Public Health and Human Services. (2016, June 3). Provider Locator Search. https://mtaccesstohealth.acs-shc.com/mt/general/providerLocator.do Accessed 14.6.2016.

Montana Department of Public Health and Human Services. (n.d.). Dental. Retrieved from http://dphhs.mt.gov/MontanaHealthcarePrograms/Dental/ Accessed 3.5.2016.

Moulavi, D., Bushy, A., Peterson, J., \& Stullenbarger, E. (1999). Factors to consider when buying a mobile health unit. Journal of Nursing Administration, 29(2), 34-41.

Ronald McDonald Care Mobile. (n.d.). http://www.svh-mt.org/services-and-departments/pediatrics-stvincent-childrens-healthcare/for-parents/ronald-mcdonald-care-mobile/ Accessed 22.7.2016.

Rural Health Information Hub. (n.d.). Health and Healthcare in Frontier Areas. https://www.ruralhealthinfo.org/topics/frontier Accessed 13.1.2017.

Sefair, J. A., Molano, A., Medaglia, A. L., \& Sarmiento, O. L. (2012). Locating neighborhood parks with a lexicographic multiobjective optimization method. In Community-Based Operations Research (pp. 143-171). Springer New York.

Skillman, S. M., Doescher, M. P., Mouradian, W. E., \& Brunson, D. K. (2010). The challenge to delivering oral health services in rural America. Journal of Public Health Dentistry, 70(s1), S49S57. 
US Department of Health and Human Services. (2000). Oral Health in America: A Report of the Surgeon General. Rockville, MD: US Department of Health and Human Services, National Institute of Dental and Craniofacial Research, National Institutes of Health, 2000. NIH Publication no. 00-4713.

US Department of Health and Human Services. (2004). The 2004 Report to the Secretary: Rural health and human services issues.

US Census Bureau. (2012). Montana 2010. https://www.census.gov/prod/cen2010/cph-2-28.pdf Accessed 1.4.2016.

US Census Bureau. (n.d.). Resident Population Data. http://www.census.gov/2010census/data/apportionment-dens-text.php Accessed 1.4.2016.

US Census Bureau. (n.d.). Geographic Terms and Concepts - Place. Retrieved from https://www.census.gov/geo/reference/gtc/gtc_place.html Accessed 1.4.2016.

US General Services Administration (2016). Per Diem Rates Look-Up. https://www.gsa.gov/portal/category/100120 Accessed 1.11.2017.

Vargas, C. M., Yellowitz, J. A., \& Hayes, K. L. (2003). Oral health status of older rural adults in the United States. The Journal of the American Dental Association, 134(4), 479-486. 